\title{
Electric current sensors: a review
}

\author{
Pavel Ripka, Czech Technical University in Prague, Czech Republic
}

\begin{abstract}
The paper makes a brief overview of traditional methods of measuremet of electric current and shows in more detail relatively new types of current sensors. These include Hall sensors with field concentrators, AMR current sensors, magnetooptical and superconducting current sensors. The influence of the magnetic core properties on the error of the current transformer shows why nanocrystalline materials are so advantageous for this application. Built-in CMOS current sensor are important tools for monitoring of the health of integrated circuits. Of special industrial value are current clamps which can be installed without breaking the measured conductor.

Parameters of current sensors are also discussed, including geometrical selectivity. This parameter specific for current sensors means the ability to suppress the influence of currents external to the sensor (including the position of return conductor) and also suppress the influence on the position of the measured conductor with respect to the current.
\end{abstract}

Key words

Hall current sensor, current transformer, AMR current sensor, current shunt, ammeter, Rogowski coil, current comparator, CMOS current sensor, magnetooptical current sensor, SQUID current sensor, GMI, current clamps

\section{Introduction}

Precise DC and AC current sensors are required by the automotive industry and by the chemical industry for measuring power and energy, and in many other applications. We discuss the principles of these devices with special reference to recent advances. We will concentrate on factors influencing precision, geometrical selectivity and other parameters important for new applications, e.g. inverters for power mills, intelligent buildings and big technological or physical experiments, e.g. electron beam measurement in the ATLAS experiment at CERN .

Classical current sensors were reviewed in [Iwansson 1999]. Most contactless current sensors are based on magnetic field sensors - these were reviewed in [Ripka 2001], and more recently in [Edelstein 2007].

The latest review of current sensors provides an excellent overview of the principles and properties of industrial devices available on the market [Ziegler 2009a]. In the present paper we concentrate on the latest research developments and laboratory devices.

\section{Overview of current measurement}

\subsection{Definition and standards}

Electric current is caused by movement of charge, and in the older MKS system it was therefore naturally defined as a derived unit. In the present sI unit system, the ampere is the fundamental unit [BIPM]. The early (1908) definition of an ampere was electrolytic. This was replaced in 1948 by a force definition. Using this definition, the ampere can be realized using the current balance. The most precise definition is the Watt current balance, which is also used to realize the "electronic kilogram": a relative uncertainty of $3.6 \times 10^{-8}$ was achieved by the U.S. National Institute of Standards and Technology (NIST) [Steiner 2007]. A much more practical realization of the ampere is via Ohm's law from the volt and the ohm, 
which are easily reproducible using the Josephson junction and the quantum Hall effect [BIPM, Appendix 2].

The proposed new definition of the ampere is a return to the natural concept based on elementary charge and time.

National metrology institutes offer calibration of current sensors with minimum expanded uncertainty (a coverage factor of 2, i.e. for 95\% level of confidence) of typically $10 \mu \mathrm{A} / \mathrm{A}$ for DC currents and $100 \mu \mathrm{A} / \mathrm{A}$ for AC currents. The AC current ratio is calibrated with minimum expanded uncertainty of 5 ppm in ratio error and 5 urad in phase displacement.

\subsection{Current shunts}

The disadvantages of current shunts are obvious: the measured current has to be interrupted to introduce the sensor, shunts for large currents are bulky, they dissipate heat and the output is galvanically connected with the measured circuit. However current-sensing resistors are a robust and cheap solution for many applications. Self-made coaxial current shunts can easily be made from a set of metal resistors in parallel [Filipski 2006, 2008].

If precision is not a critical consideration, the current can be measured even through the voltage drop across a current-carrying copper trace. A microcontroller was used to calibrate the copper trace resistance and implement temperature drift compensation by means of a temperature sensor [Ziegler 2000c]. Corrections should be made for the thermal resistance between the busbar and the temperature sensor. The authors have shown that the dynamic properties of this sensor are not due to the self-inductance of the busbar, but due to the mutual inductance between the main loop and the sense loop.

In order to measure very small currents, the value of the current sensing resistor should be increased so that the measured drop is measurable. This leads to problems. A systematic error is caused by a divider consisting of the source internal resistance and the sensing resistor. High value resistors have poor time and temperature stability, they are noisy and, together with the input capacitance of the voltage amplifier, they create a large time constant which may slow down the measurement.

The noise sources are shown in Fig. 1.

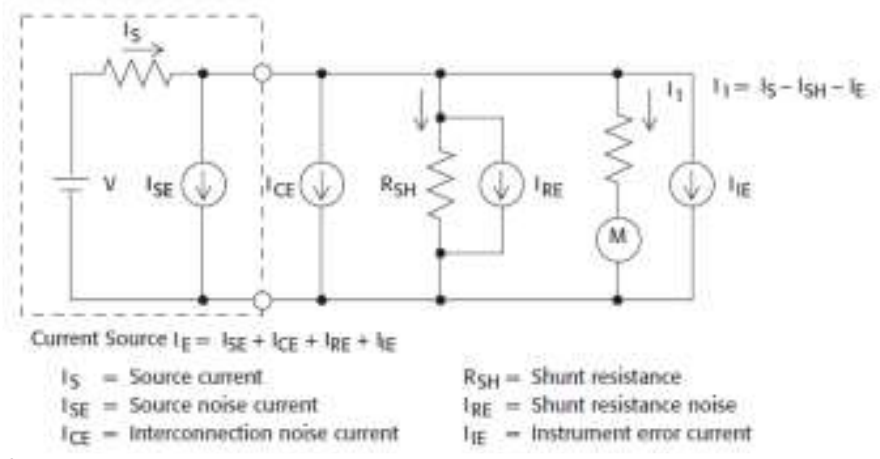

Fig. 1.Sources of current errors - from [Keithley 2007]

The noise current generators $I_{\mathrm{CE}} \mathrm{I}_{\mathrm{SE}}$, and $I_{\mathrm{IE}}$ in the above model represent unwanted currents generated at a particular point in the circuit. These currents may arise from triboelectric, piezo-electric and electrochemical effects, or from resistive leakage or dielectric absorption (Fig. 3). 


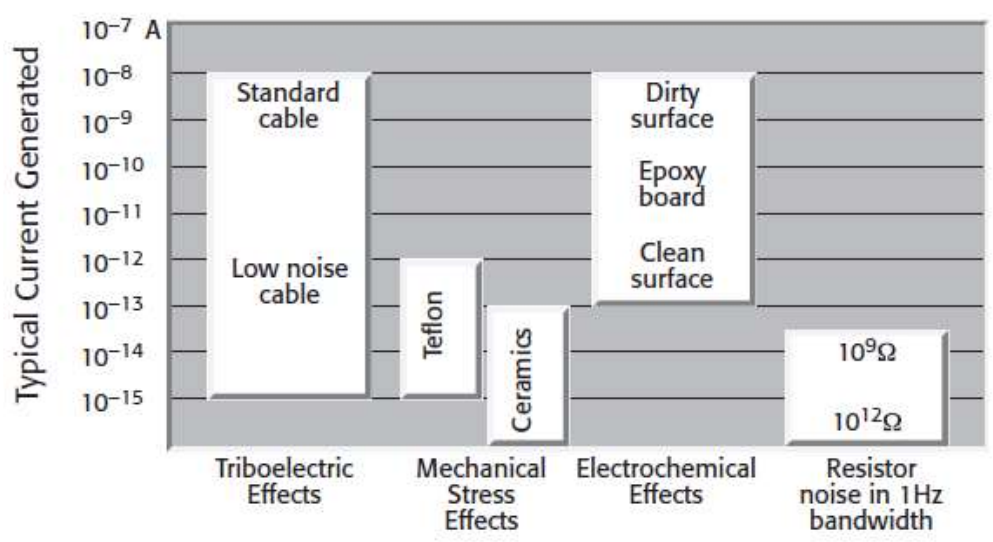

Current-Generating Phenomena

Fig. 2 Typical magnitudes of currents generated by low current phenomena - from [Keithley 2007].

For measuring picoamps and lower currents, a current-to-voltage configuration is preferred.

\subsection{Current-to-voltage converters}

This circuit is also referred to as a feedback-type ammeter (Fig. 3)

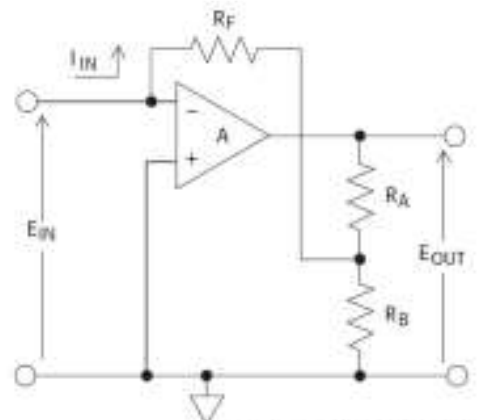

Fig. 3 Current-to-voltage converter - from [Keithley 2007]

The range is changed by output divider $R_{A}$, $R_{B}$ rather than by changing the feedback resistor $R_{F}$. Supposing that $R_{F}>R_{A}$, $R_{B}$ we may write for the output voltage:

Vout $=-\operatorname{IR}_{\mathrm{F}}\left(1+R_{\mathrm{A}} / \mathrm{R}_{\mathrm{B}}\right)$

The parasitic capacitance of the feedback resistor $\mathrm{CF}$, which limits the speed, can be effectively compensated by the circuit shown in Figure 4. If time constant $R_{1} C_{1}$ is made equal to time constant $R_{F} C_{F}$, the shaded area of the circuit behaves exactly like resistance $R_{F}$ with zero $C_{F}$. The matching of the time constants in this case is fairly straightforward, because the capacitances involved are all constant and are not affected by the input capacitances [Keithley 2004]. 


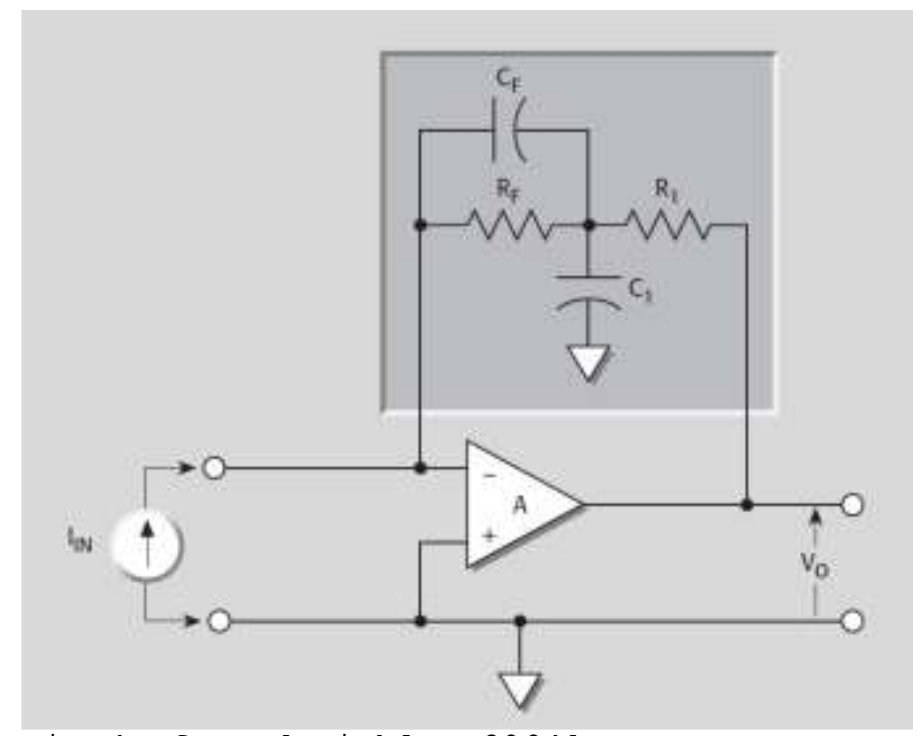

Fig 4 from [Keithley 2004].

A simple current-to-voltage converter with an amplification factor up to $1 \mathrm{~V} / \mathrm{nA}$ can be built using off-the-shelf operational amplifiers. A converter based on OPA129 (Texas Instruments) has a $100 \mathrm{fA}$ bias current [Weckenmann 2008]. Other picoammeters are based on AD549L (Analog devices) with a bias current of $40 \mathrm{fA}$ and noise of $0.36 \mathrm{fA} \mathrm{p}-\mathrm{p}$ in the $0.1 \mathrm{~Hz}$ to $10 \mathrm{~Hz}$ bandwidth [Northrop 2005]. The noise has a $1 / \mathrm{f}$ character, so $0.1 \mathrm{fA} / \sqrt{H z} @ 1 \mathrm{kHz}$ increases to $10 \mathrm{fA} / \sqrt{\mathrm{Hz}} @ 0.1 \mathrm{~Hz}$. These are extraordinary values compared to the $4 \mathrm{fA} / \sqrt{ } \mathrm{Hz}$ noise of superconducting current amplifiers [Gay 2000].

It is sometimes easier to measure small currents by integrating them (using a coulomb meter). In this case, the feedback resistor is replaced by an integrating capacitor, which is theoretically noiseless. The charge method is preferable when current resolution below $1 \mathrm{fA}$ is required, as values of $R_{f}>10^{12}$ are very impractical.

The achievable current resolution of available ammeters is $10 \mathrm{fA}$. Smaller currents can still be measured with conventional instruments, but the measurement is slow in order to average out the instrument noise. Superconducting current sensors may be much faster, but the fundamental speed limit of all current measurements is set by the fact that 1 aA corresponds to 6 electrons/sec.

\section{Current sensor parameters}

The basic parameters of any sensor are linearity, offset (for DC sensors) and sensitivity, and also stability of offset and sensitivity with temperature and time. Also important is the sensor bandwidth (of more generally frequency characteristics, as current waveforms often contain high frequency component. Current sensors which contain ferromagnetic material also suffer from hysteresis and perming (change in offset after the sensor is subjected to the large overcurrent from a magnetic field shock). It is also important for contactless sensors to be insensitive to the actual position of the measured conductor and also resistant to external currents and magnetic fields. We call this parameter "geometrical selectivity", and consider it to be critical for most applications. The most common way to achieve geometrical selectivity is to use a closed magnetic circuit with a measured conductor inside. This is used in current transformers, fluxgate current sensors and in most Hall current sensors. The high-permeability magnetic circuit (the yoke is often in the shape of a ring) concentrates all field lines from the measured current, so that the measured flux in the magnetic circuit does not depend on the actual position of the conductor. In thiscase, $\mathrm{H}$ in the yoke central line $l$ is constant, and we can write

$$
\mathrm{HI}=\mathrm{NI} \text {, }
$$

where $I$ is measured current and $N$ is number of turns. The magnetic circuit also shields against external fields. It is important to use a high cross-sectional 
area of the magnetic material to keep a high demagnetisation factor against external fields.

The magnetic yoke should not be saturated, i.e. $1>\mathrm{NI} / \mathrm{H}_{\text {sat }}$. This means that the magnetic circuit becomes very large for high measured currents. Airgaps can be introduced, but these degrade the geometrical selectivity. Another approach is to compensate the measured current using a multi-turn coil. However, generating the compensation current requires power.

Magnetic gradient techniques can be used if the power or space limitations do not allow the use of a yoke. Simple integrated current sensors use a folded conductor and a gradient field sensor. This suppresses the response from distant sources, giving a low gradient. For large currents, the current bar should be kept straight, and circular arrays of typically four to eight sensors are used [Di Rienzo 2003]. Averaging the sensor output increases the sensitivity to the conductor between them and decreases sensitivity to conductors outside. Instead of simple averaging of the sensor outputs, more sophisticated signal processing methods have been developed to suppress the effects of nearby false currents more effectively [Bazzocchi 2000].

The best magnetic circuit for a current sensor is a toroid made from high permeability material without an airgap. This is used in current transformers and in some residual current sensors. However, even a toroid cannot be considered as ideally homogeneous: as it is often wound from tape material, and the beginning and end of the strip cause significant asymmetry. Another problem may arise from the presence of airgaps between the layers. They may cause concentration of the flux to the first layer, which can be saturated. These errors are not only of concern in the case of precise sensors, such as current comparators - they may cause abnormal tripping of ordinary current breakers [Colin 2006].

Wide bandwidth: a large frequency range from DC to several $\mathrm{MHz}$ is required for applications in switching power converters.

High dynamic range and resistance against overload is another important parameter. Accuracy is often required even for a small fraction of the fullscale. One example is the requirement to measure the current consumption of the house in the standby mode and during full operation.

Immunity against $\mathbf{d v} / \mathbf{d t}$ is critical for use in modern power modules. Current sensors located close to fast-switching transistors can be subjected to transients of $10 \mathrm{kV} / \mu \mathrm{s}$. Such transients can be coupled by parasitic capacitances to the output of the current sensor. Proper electrostatic shielding can efficiently suppress this effect, but it complicates the design of the sensor. Low cost and high environmental resistance is a must for industrial sensors.

\section{Applications and demands}

Current sensors are required for numerous applications, including

- Current leakage monitoring.

- Industrial applications - large AC and DC currents.

- Car applications: special requirements for hybrid cars.

- House automation.

- Energy production, conversion and storage

- Motor drives, power converters and power modules

- Current sensors embedded in ICs

We only briefly mention specific issues in connection with the last two applications

\subsection{Power converters and power modules}

Current sensors are essential for the control of power converters. They are normally used for current programming and overcurrent protection. Input and output voltages are usually used for the control. In the case of galvanically insulated converters, optocouplers are often used to insulate one of these voltages. Limited linearity of optocouplers can be a problem in converters with wide variation of output, e.g. DC/AC converters. Qiu [2003] has shown that the whole converter can be controlled using only current information, so that voltage 
sensors are not necessary. This approach can simplify the design of galvanically insulated power converters.

Bai [2003] suggests the use of magnetooptical current sensors for applications in integrated power modules. The most promising candidates are sensors using rareearth iron garnet sensing material between two optical fibres. Although the concept was proven to work, it is at present not cost-effective.

\subsection{Built-in current testing and embedded sensors}

The built-in CMOS current sensor (BICS) was designed to monitor the current consumption $\left(I_{D D}\right)$ of integrated circuits, as it is a simple method for detecting defects missed by conventional logic tests [Cimino 2007].

The sensor uses a parasitic $10 \Omega$ resistor in the IC interconnecting layer; the effect of the process parameter variation is reduced by a ratiometric circuit. The circuit is able to measure $20 \mathrm{~mA}$ current with $0.5 \%$ accuracy. Although the measured current is reduced by a factor of 10 using a current mirror, the sensor consumes $2 \mathrm{~mW}$ power, which may be unacceptable for some applications. A possible solution is to employ a switching structure which bypasses the current-to-voltage conversion transistors for most of the operational time, and performs the test only on demand. This allows a current sensing structure to be built with power consumption in the order of $\mu \mathrm{W}$, which does not cause significant speed degradation of the measured circuit [Kim 2006]. Another way is to use dynamic switching: the switch between the supply voltage and the circuit under test (CUT) is open, and the decaying voltage is observed. The time that the voltage takes to drop to the reference level is measured by the counter. Very fast measurement with $1 \mu \mathrm{A}$ resolution is achievable using this technique [Vazquez 2004].

The radiation sensitivity of VLSI devices has become an important issue, as these circuits are sensitive not only to cosmic rays, but also to the atmospheric neutrons and protons present at low altitudes and to natural alpha particles. Bulk BICS can detect the transient currents generated by the impact of an energetic radioactive particle at a sensitive circuit node of an integrated circuit [Wirth 2008]. Donoval [2007] suggested the use of a MAGFET transistor for embedded current monitoring, but this research is at the very beginning.

The current sensor structure can be embedded into the lateral insulated-gate bipolar transistor (IGBT) structure. 5\% accuracy is achievable over a wide temperature range, which is sufficient for overload and short-circuit protection [Liang 2003].

\section{Contactless sensor principles}

Current measurement using a shunt resistor is impractical or impossible in some cases. Contactless current sensors keep galvanic insulation between the measured current and their output voltage. A wide range of AC and DC contactless current sensors are produced by LEM, F.W. Bell, VAC, Honeywell, Telcon and many other manufacturers. Magnetic field sensors can be used to measure currents in remote conductors at high potentials, in underground cables and in building structures.

\subsection{Instrument current transformers}

A current transformer (CT or ICT) usually has a bulk ring core made of highpermeability material. For currents higher than 50 A the primary winding is usually a single conductor through the core opening. The secondary winding should ideally be short-circuited; however for most applications it is connected to a small 'burden' resistor or impedance. The core is wound with high permeability tape (for low-frequency devices) or is made from ferrite (for high-frequency current probes). In some devices, the secondary current is measured by active current-to-voltage converted in such a way that the burden is virtually zero. Later we will show that this leads to smaller error, as the transformer core works at lower flux density.

Current transformers are also made with an openable core, most often as AC current clamps for oscilloscopes or multimeters.

Current transformers are very popular devices:

- they are very simple and robust 
- they do not require external power

- they have high galvanic insulation

- they are cheap

- they have a long lifetime with invariant parameters

CTs should be periodically calibrated. This is usually performed using a standard CT and a current comparator (Chapter 4.4.) Fully automated transformer measurement sets are available on the market (e.g. Tettex 2767).

National metrology laboratories usually calibrate current transformers with a nominal secondary current of $5 \mathrm{~A}$ or $1 \mathrm{~A}$, with test points of $(1 ; 5 ; 20 ; 100$ \& 120) \% of rated primary current. Routine tests are performed at 50, 60 and $400 \mathrm{~Hz}$ with uncertainties of $0.01 \%$ in ratio error and 0.1 mrad in phase angle displacement. Less accurate on-site calibration can be carried out using a Rogowski coil and a coaxial shunt resistor [Suomalainen 2009].

Calibration equipment based on current comparators is bulky and expensive. Brandolini [2009] proposed a novel method based on digital processing of signals that are directly collected at the secondaries of the transformers under test and a reference transformer. The errors of the proposed system are also almost constant for an input signal with a frequency of up to $1 \mathrm{kHz}$. When compared to Tettex 2767, the achieved uncertainty at $50 \mathrm{~Hz}$ was below $0.013 \%$ in amplitude and $0.16 \mathrm{mrad}$ in phase. At higher frequencies up to $1 \mathrm{kHz}$, the error was below $0.025 \% / 1.3 \mathrm{mrad}$. This means that even if this instrument does not achieve the precision of a current comparator, it can be used to calibrate a 0.1 precision class electronic measurement transformer, as defined by the IEC 60044-7 and IEC 60044-8 standards.

Fig. 5 shows the equivalent circuit of the current transformer for low and medium frequencies

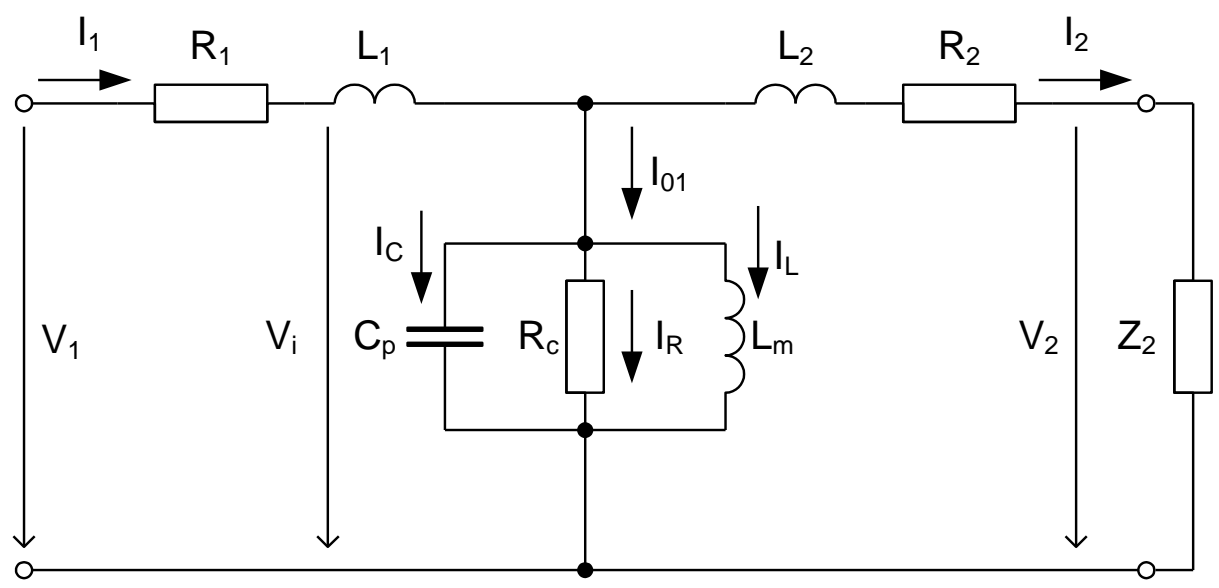

Fig. 5 after [Draxler 2007]

$R_{1}, R_{2}, L_{1}$ and $L_{2}$ represent resistances and leakage inductances of primary and secondary windings,

$R_{c}$ is the resistance representing losses in the ferromagnetic core,

$\mathrm{L}_{\mathrm{m}}$ is the magnetizing inductance,

$\mathrm{C}_{\mathrm{p}}$ represents the parasitic capacitances of the winding,

$Z_{2}$ is the burden

and $I_{L}+I_{R}$ is the magnetization current

This equivalent circuit is valid for a 1:1 current ratio. For other current ratios, the circuit parameters can be recalculated either to the primary side or to the secondary side. Currents and voltages are recalculated by the turn number ratio $\mathrm{N}$, and impedances are recalculated by $\mathrm{N}^{2}$.

At low and medium frequencies, the influence of parasitic capacitances and leakage inductances can be neglected, and a simplified phasor diagram according to Fig. 6 can be used. 


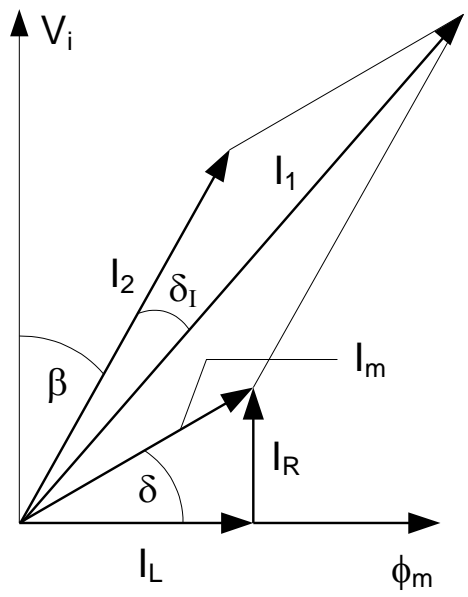

Fig. 6 after [Draxler 2007]

$I_{m}$ is magnetizing current of $C T$, consisting of components $I_{R}$ and $I_{L}$,

$\Phi$ is magnetic flux of core,

$\delta$ is loss angle of the ferromagnetic core,

$\delta_{I}$ is CT phase displacement, and

$\beta$ is the phase displacement of the secondary burden (which is often negligible).

For a properly working CT the phase error $\delta_{I}$ is small and, according to Fig. 7, we can write for the amplitude and phase errors:

$\varepsilon_{I}=\frac{\left|\mathbf{I}_{2}\right|-\left|\mathbf{I}_{1}\right|}{\left|\mathbf{I}_{1}\right|} 100 \cong-\frac{I_{m} \sin (\delta+\beta)}{I_{1}} 100(\%)$

$\delta_{I} \cong \operatorname{tg} \delta_{I} \cong-\frac{I_{m} \cos (\delta+\beta)}{I_{1}}(\mathrm{rad})$

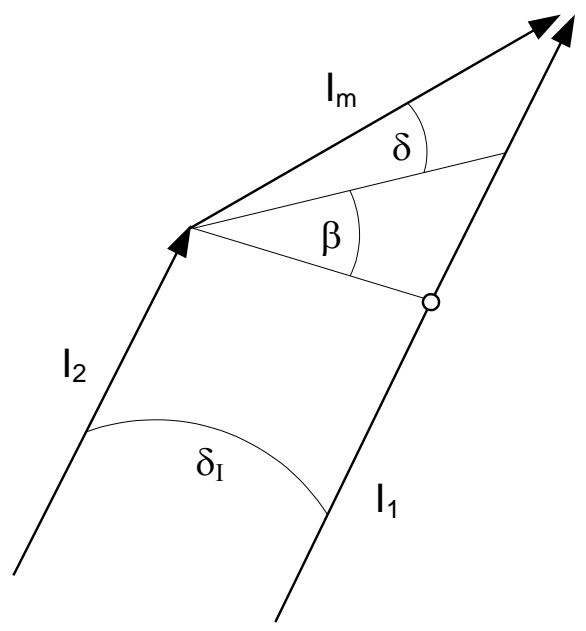

Fig. 7 after [Draxler 2007]

It is clear that in order to keep the error low at low frequencies, it is important to keep $R_{c}$ and $L_{m}$ high and also to keep $R_{2}$ and $Z_{2}$ small. The magnetizing inductance $\mathrm{L}_{\mathrm{m}}$ is given by 
$L_{m}=\frac{\mu_{r} \mu_{0} N^{2} A_{C}}{l_{C}}=\frac{\mu_{r} \mu_{0} N^{2} A_{C}}{2 \pi r}$

where $r$ is the mean radius of the toroidal core

and $A_{c}$ is the cross-sectional area of the core.

$L_{m}$ is kept high when using a high permeability core with a high number of turns. As the inductance depends on the square of the number of turns $\mathrm{N}^{2}$ and the wire resistance depends only on $\mathrm{N}$, a 1:5 turn ratio gives a larger error than 10:50. By increasing the number of turns we can bring the core to saturation: this should be avoided by using a higher-diameter ring. The main inductance also depends linearly on the cross-sectional area of the core. For this reason, precise CTs are large.

At low frequencies (such as $50 / 60 \mathrm{~Hz}$ ), the dominant source of error is the magnetizing current, which is inversely proportional to the frequency. The following methods can be used to reduce this current:

- use a core material with high permeability

- increase the core area

- increase the number of turns

- virtually increase the core permeability by a feedback amplifier (used in self-balancing current comparators)

The magnetizing inductance $\mathrm{L}_{\mathrm{m}}$, together with secondary resistance $\mathrm{R}_{2}$ and burden resistance $\mathrm{R}$, form a high-pass filter circuit. Another approach to improve transformer accuracy at low frequencies is therefore to decrease the burden resistance $\mathrm{R}$, which can be done by using a current-to-voltage converter [Kondrath 2009].

Some CTs have almost a constant ratio error for a wide range of measured currents. In this case, the error can be compensated for by adding extra turns to the secondary winding. This is called turn error compensation. Transformers with such compensation show anomalous behaviour - their error increases when the burden decreases. Magnetization of the CT core (e.g. due to a lightning strike) may also lead to increased errors when the measured current is small so that the $\mathrm{CT}$ core is not demagnetized [Draxler 2007].

\section{Electronically enhanced current transformers}

The first step is to connect the output of the CT to a current-to-voltage converter with a very small input resistance. This will minimize the burden, and it keeps the flux in the CT core at a low level, but not at zero - this is clear from the equivalent circuit of the CT in Fig. 5. In order to achieve really zero flux, it is necessary to keep $V_{i}=0$, and not only $V_{2}=0$. This can be achieved by a simple analog circuit [Baccigalupi 2009]. The test was performed with a lowcost CT with a ferrite core: at $50 \mathrm{~Hz}$ and also for higher frequencies up to 450 $\mathrm{Hz}$ the ratio error was reduced from 2\% to $0.7 \%$. The phase error was reduced from $45 \mathrm{mrad}$ to $3 \mathrm{mrad}$ at $50 \mathrm{~Hz}$, but this error increased linearly with frequency due to the delay in electronic circuits. It should be noted that this type of enhancement does not work properly for CT with turn error compensation.

Electronically enhanced two-stage current transformers show accuracy improvement by two orders of magnitude: with a low burden, the resulting error is below 10 ppm [Miljanic 1991]. These devices can also indicate the remanence of the transformer core or DC component in the primary current, which may degrade the performance of a classical current transformer; they may have a lower number of turns, which avoids problems with parasitic capacitances and enables the device to be used at higher frequencies; and finally the volume of the core may be reduced. 
Amplifier-aided wide-band two-stage current transformers may have 1 ppm accuracy from $50 \mathrm{~Hz}$ up to $1 \mathrm{kHz}$, and $15 \mathrm{ppm}$ accuracy up to $10 \mathrm{kHz}$ [Souders 1972].

At higher frequencies, parasitic capacitances between the turns and layers of the winding are the dominant source of error. Here, increasing the number of turns increases the error. For precise fast-current transformers, amorphous cobaltbased cores or ferrite cores are used, which give high permeability at $\mathrm{MHz}$ frequencies. Such transformers are used to observe short beam pulses in particle accelerators. [Bergoz] manufactures transformers with cut-off frequencies up to 2 $\mathrm{GHz}$.

A high-frequency model of a current transformer is given in Fig.8 [Kondrath 2009]. It is shown that the effect of $L_{m}, R_{1}$ and $L_{1}$ can be neglected. We also neglect the inductance of the sensing resistor. The frequency bandwidth is usually given by the first pole $\mathrm{f}_{\mathrm{p} 1}=1 / 2 \pi \mathrm{R}_{2} \mathrm{C}$. A second pole, defined by stray inductance $\mathrm{f}_{\mathrm{p} 2}=\mathrm{R}_{\mathrm{c}} / 2 \Pi \mathrm{L}_{2}$, is normally at a much higher frequency.

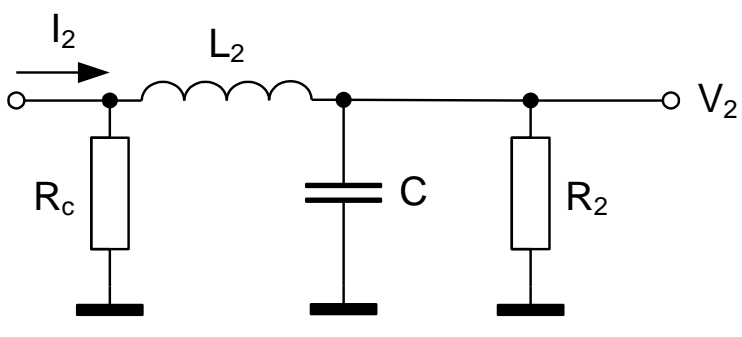

Fig. 8 High-frequency equivalent circuit of the current transformer - after [Kondrath 2009].

There have also been efforts to digitally compensate the frequency dependent errors of current transformers. If the current transformer is considered to be a linear system (which is often true for low burdens), its frequency characteristics can be identified and corrected using a frequency filter with inverse characteristics. An efficient way to build this type of filter is with the use of FPGA (Field-programmable gate array). Both the amplitude and the phase error of a low-cost current transformer with an accuracy class of 0,5 was reduced by a factor of 20 [Gallo 2009]. Such a solution can be cost-effective, due to the very low cost of digital hardware. A disadvantage is that digital electronics introduces an inevitable time delay. If this cannot be eliminated by postprocessing, it will lead to large, frequency-dependent phase error.

Current transformers are often used in electronic watt and energy meters. For this application, it is very important to make them resistant against saturation - either from a strong permanent magnet, or due to a DC component in the measured current [Mlejnek 2009]. Possible methods for avoiding such saturation are: 1. use flat-loop magnetic material, which has a very high saturation field H 2. use composite cores, consisting of a high-permeability ring for precision and a low-permeability ring for saturation immunity

3. detect saturation by other means

4. use a Rogowski coil instead of a CT, which has no magnetic core

The composite high-permeability core gives low angular and amplitude error for most applications, but this is not guaranteed for all types of loads. Flat-loop materials such as Vacuumschmelze VAC do a better job, but these materials are expensive. It was recently shown that some cheap low-permeability rings can also be used for this application: they have a relatively large phase shift, but it is constant over the wide range of measured currents and can therefore be compensated [Mlejnek 2007, 2009b].

Fe-based nanocrystalline alloys are promising materials for very precise smallsize current transformers [Draxler 1996]. They may have the same permeability as permalloys (NiFe crystalline alloys) or Co-based amorphous alloys, but they have 
much larger saturation induction, so that instr)ument current transformers are smaller. Properly prepared Fe-based nanocrystalline ring-cores may have almost constant permeability over a wide range of induction. This gives a low amplitude error and a constant phase error in a wide range of the measured current. Such a constant phase error can easily be compensated. In large amounts, Fe-based nanocrystalline materials can be purchased at competitive prices.

\subsection{Rogowski coil}

The Rogowski coil for measuring current is an air coil wound around the measured current conductor. The basic operating principle is given by the mutual inductance $M$ between the primary (single turn) and the secondary (many turns). The output voltage is proportional to the derivative of the current:

$$
\mathrm{u}=\mathrm{M}(\mathrm{dI} / \mathrm{dt})
$$

The coil should be precisely manufactured with constant winding density and diameter. Ideally, a homogeneous coil has excellent geometrical selectivity, i.e. it is insensitive to external fields and to the position of the measured conductor), as it follows Ampere's law in the open air:

$$
\oint_{C} \vec{B} \cdot d \vec{l}=\mu_{0} i_{C}
$$

where the integration path $\mathrm{C}$ is the central line of the coil (usually a circle).

In order to obtain the AC current waveform, a Rogowski coil is used together with an integrator. Single-chip digital integrators have been developed to process the signal of Rogowski coils (also known as dI/dt sensors) for energy meters. The use of an $\mathrm{AD} 7759$ signal processor with built-in sigma-delta A/D converters gives 0.1\% error from the measured value in the 1000:1 dynamic range [Koon 2009].

The Rogowski coil contains no ferromagnetic material, and thus it has excellent linearity and an extremely large dynamic range. Users often rely on their linearity and use them to measure currents that are much higher than the currents used for calibration. Care should be taken with the geometry of the connecting bus bars and the return conductor to reduce the effect of magnetic coupling. The Rogowski coil can be very simply temperature compensated. With increasing temperature the coil former expands, causing about $50 \mathrm{ppm} / \mathrm{K}$ temperature coefficient of the scale factor. However, the resistance of the copper coil increases with a 3900 ppm/k temperature coefficient. By connecting a proper lowtemco resistor in parallel to the Rogowski coil, we create a voltage divider which compensates the thermal expansion. The resulting temperature coefficient can be as low as $2 \mathrm{ppm} / \mathrm{K}$ [Suomalainen 2009].

Stationary Rogowski coils are used to measure AC or transient currents or changes in DC currents. For long-term measurements, the limiting factor is the offset drift of the integrator. It is also possible to measure DC current with an openable Rogowski coil: the output voltage is integrated while the coil is closed around the measured conductor.

Flexible Rogowski coils can be easily wound on top of a plastic cable with thick insulation, such as a coaxial cable with removed shielding. The inside conductor is used as a return loop, which compensates the perpendicular virtual loop created by the winding advance. An uncompensated loop would cause sensitivity to external magnetic fields in the axial direction. A detailed description of the production and testing of such coils and the following integrator and filter can be found in [Abdi-Jalebi 2007]. The error caused by the off-center position of the measured current was below 1.5\%, and the suppression of the external currents was better than 100 . 


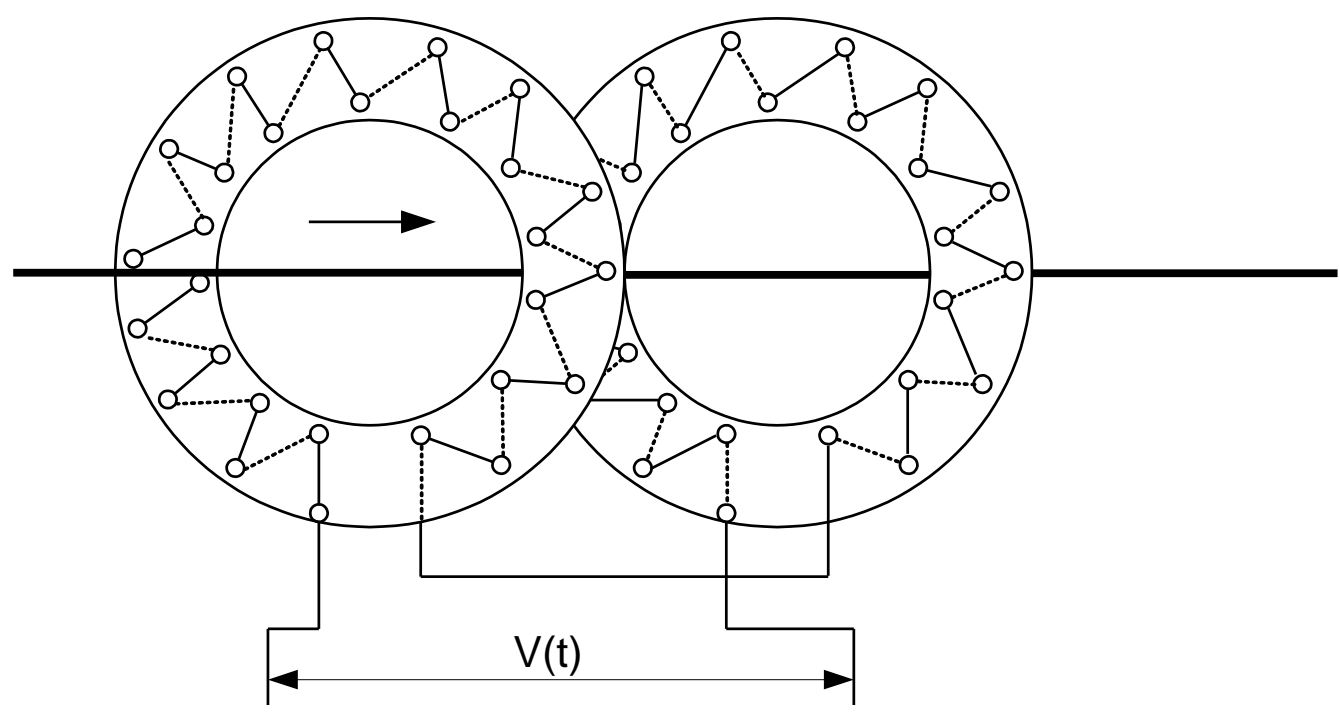

Fig. 9 A Rogowski coil in PCB technology. Each layer is wound in the opposite direction to compensate for the loop created by turn advancement after Kojovic 2002

Rogowski coils are useful for measuring transient currents. The coil selfinductance and the parasitic capacitance form a resonant circuit. In order to increase the resonance frequency of this circuit, a lower number of turns should be used, which decreases the sensitivity. 1 to $3 \mathrm{MHz}$ resonance frequency is typical for flexible coils, while multiturn solid coils may have resonance as low as $50 \mathrm{kHz}$. At high frequencies, the active integrator can also cause errors due to the limited bandwidth and slew-rate of the amplifier. An alternative technique for extending the frequency range is to use the current output instead of integrating the output voltage [Ward 1993].

Printed circuit board (PCB) technology was used to produce Rogowski coils with a more precise geometry and improved temperature stability. To compensate the virtual perpendicular loop, two PCB coils with opposite winding directions are connected in series. In order to achieve perfect compensation it is important to design these two coils with the same diameter, as shown in Fig. 9.

An improved twin-loop PCB Rogowski coil with a transfer ratio of $4 \mathrm{~V} / 400 \mathrm{kA}$ was used to measure the plasma current in the Tokamak. Thanks to good homogeneity of the winding, the change in the transfer ratio with the geometrical location of the measured current inside the measuring channel is less than $2 \%$. This is very important for this application, as the exact location of the plasma current in the $1 \mathrm{~m}$ diameter vacuum vessel is unpredictable [Qing 2009]. In this design, both coils are made in the same place, which means that one of them has a smaller diameter and thus the sensitivity to axial fields is not perfectly compensated.

Bull [2005] presented a hybrid Rogowski current sensor for high-voltage applications. The device consists of a Rogowski coil with a passive integrator connected to an Integrated-Optic Pockels Cell (IOPC). This passive cell was developed for electric field sensing. Here it is used to modulate the light brought in and out by two optical fibres. The high-voltage part requires no electricity supply. The device has $0.3 \%$ accuracy for currents up to $30 \mathrm{kA}$ in the range from -30 to $+70^{\circ} \mathrm{C}$.

\subsection{DC current transformers and current comparators}

Classical current transformers naturally measure only AC currents. DC current transformers and DC current comparators (Section 4.4) use the fluxgate effect in a core which is periodically saturated by the excitation field. If no DC current is present, the core flux and also the voltage induced into the detection winding contain only odd harmonics. DC current shifts the magnetization characteristics, and the flux waveform is no longer symmetrical. This induces even harmonics proportional to the measured DC current. The first sensors of this type were transductors [Bera 2003]. Modern DC current transformers use phase-sensitive detectors to extract the second harmonics from the induced voltage. 
The accuracy of a typical commercial 40 A DC current transformer is $0.5 \%$, linearity 0.1\%, current temperature drift $<30 \mathrm{~mA}\left(-25^{\circ} \mathrm{C} .70^{\circ} \mathrm{C}\right)$ [Ripka 2004]. A self-oscillating sensor of this type is described by Ponjavic and Duric [2007]. The first fluxgate current sensor in PCB (printed circuit board) technology was described in [Belloy 2000]. Its sensor had a single winding of 36 turns over a toroidal core made of amorphous magnetic tape. It achieved $10 \mathrm{mV} / \mathrm{A}$ sensitivity and ranges up to $5 \mathrm{~A}$. A prototype of a fluxgate current sensor with an electroplated core in PCB technology was described in [Ripka 2005]. Short excitation pulses should be used in order to lower the excitation power. This can be achieved using a tank circuit with an external saturable inductor [Tang 2004]. Another geometry for a pcb fluxgate current sensor with an electroplated core was used by [O'Donnell 2006].

Because of their low offset drift, fluxgate-based "DC current transformers" are superior to current sensors that have a Hall sensor in the airgap. A disadvantage is the large power consumption of these devices.

A simple integrated overcurrent sensor was described in [Fujiyama 1997]. The sensor is similar to a DC current transformer, but the excitation current does not saturate the ring core. Once the core is saturated by the measured current, the output voltage drops to zero.

\subsubsection{Current comparators}

Current comparators are described in a classical book written by Miljanic and Moore [Moore 1998].

An AC current comparator is a passive device which has three windings on a ring core: primary, secondary and detection (Fig. 10). If the primary and secondary currents are balanced, i.e. $\mathrm{N}_{1} \mathrm{I}_{1}=\mathrm{N}_{2} \mathrm{I}_{2}$, the core flux and also the voltage $V_{d}$ induced into the detection winding is zero. AC comparators serve as national standards of current ratio, and they are used for calibrating standard current transformers. Practical devices are large and complex: the differences between $\mathrm{N}_{1} \mathrm{I}_{1}=\mathrm{N}_{2} \mathrm{I}_{2}$ are compensated by additional windings $\mathrm{N}_{\varepsilon}$ and $\mathrm{N}_{\varphi}$, and they have several active and passive shieldings to reduce errors. AC comparators have errors below 1 ppm in amplitude and $3 \cdot 10^{-6}$ deg in phase.

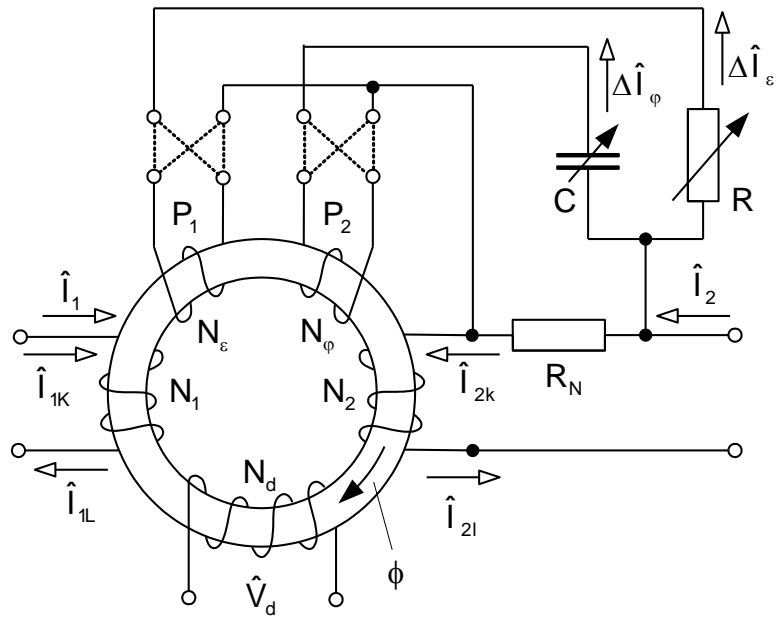

Fig. 10 AC current comparator (will be redrawn)

DC current comparators are precise devices based on the fluxgate effect. The core consists of two detection ring cores excited in opposite directions by the excitation winding $\mathrm{N}_{\text {exc }}$ supplied by current generator G (Fig. 11). The second harmonic component of the voltage induced into the detection winding $\mathrm{N}$ is measured by phase sensitive detector PSD. The output from PSD is filtered and amplified, and controls the DC compensation current $I_{2}$. In the ideal case $N_{1} I_{1}=$ $\mathrm{N}_{2} \mathrm{I}_{2}$ and the device output is derived from $\mathrm{I}_{2}$ using shunt resistor $\mathrm{R}$. Only one layer of magnetic shielding under the secondary winding is shown in the figure. The role of this shielding is twofold: it reduces the leakage fluxes originating 
from the non-homogeneity of the detection cores and the non-homogeneity of the windings, and it also provides magnetic shielding against ambient fields.

DC current comparators can have errors below 1 pm. Even more precise are cryogenic current comparators, which use superconducting shielding and a SQUID as a null detector. They are used in resistance bridges with relative measurement uncertainties of about $10^{-9}$ [Gotz 2009]. Superconducting current sensors are described in Section 4.7 .

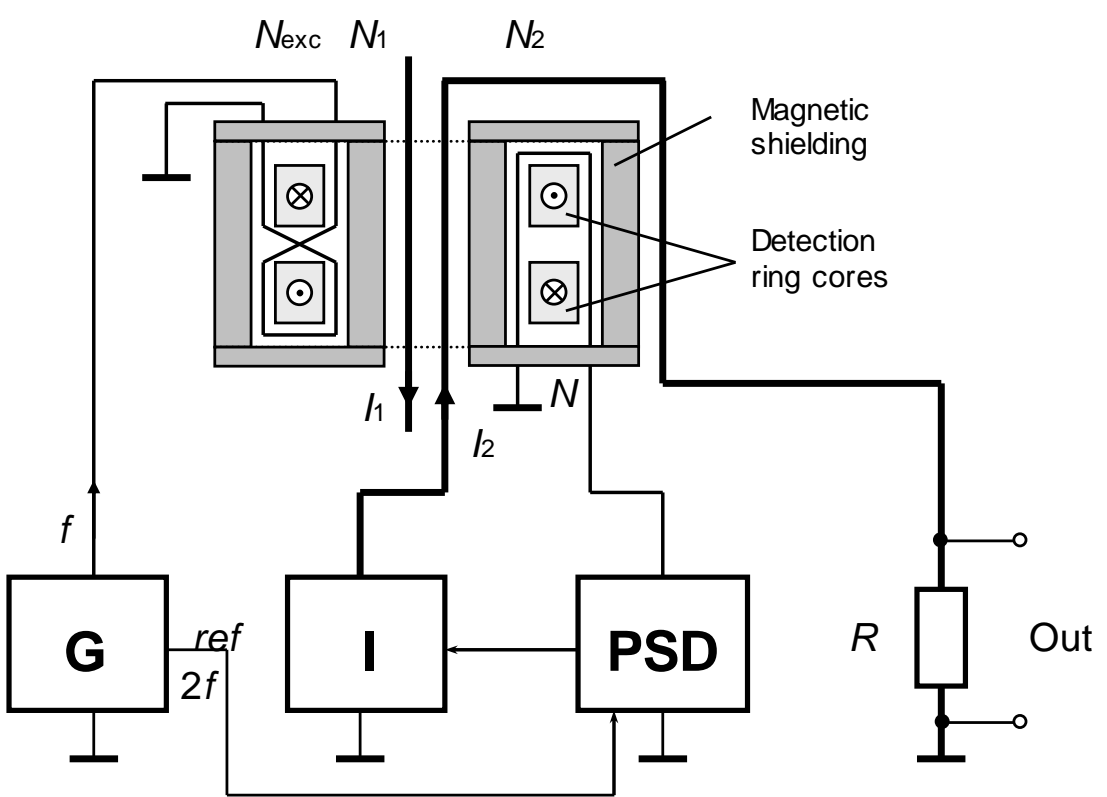

Fig.11 DC Current comparator (from [Kejik 1999])

A small-size AC/DC current comparator with amorphous cores excited in resonant mode by short 16 A p-p current pulses was described in [Kejik 1999]. The range is 200 A from DC up to $3 \mathrm{kHz}$. Depending on the applied frequency, the device works in three modes: as a passive DC comparator, a passive AC current transformer and an active feedback-compensated AC/DC comparator.

\subsubsection{Particle beam sensors}

A large bandwidth electron beam sensor works from DC up to $50 \mathrm{MHz}$ [Unser 1981]. The DC channel is made by a DC transformer, and the AC current component is measured by an active current transformer. The highest frequency components do not pass through the operational amplifier and the feedback loop - they are directly fed through a passive RC return path.

Unser [1992] describes another high-resolution current sensor developed at CERN for particle accelerators. This "Parametric Current Transformer" is a complicated device with 5 separate magnetic cores made of amorphous cobalt-based Vitrovac 6025, and several magnetic shields. The name is derived from parametric amplification, which is used in the magnetic modulator. The magnetic modulator is excited by $7 \mathrm{kHz}, 2.5 \mathrm{~A}_{\mathrm{pp}}$ current, and its output is synchronously detected at 14 $\mathrm{kHz}$. This channel measures the DC and low-frequency component of the current. The high frequency component is measured by an active current transformer. The full scale range (FS) of the instruments can be $10 \mathrm{~mA}$ to $100 \mathrm{~A}$. The achieved linearity error is $10 \mathrm{ppm}$ of $\mathrm{FS}$, the zero temperature drift is $\pm 5 \mu \mathrm{A} /{ }^{\circ} \mathrm{C}$, the resolution \pm $0.3 \mathrm{ppm}$ of $\mathrm{FS} \pm 0.4 \mu \mathrm{A}$, and the frequency band is DC up to $100 \mathrm{kHz}$. This device was later industrialized by Bergoz Instrumentation [Bergoz].

\section{$5.4 \mathrm{Hall}$ current sensors}

Many DC current sensors use a Hall element mounted in the airgap of a magnetic core (Fig. 12). The yoke has two important effects: 1 . increasing the sensitivity, 2. increasing the geometrical selectivity, i.e. shielding the 
external fields and decreasing the influence of the position of the measured current.

Even when using a magnetic yoke, Hall current sensors are sensitive to external magnetic fields and nearby currents, and also to the position of the measured conductor, due to the nonhomogeneity associated with the airgap.

A serious DC offset can be caused by the remanence of the magnetic core - only a few Hall current meters have an AC demagnetization circuit to erase perming after the sensor has been exposed to a large DC current or external field. In general, the yoke material should have large saturation induction $B_{s}$ and low coercivity $\mathrm{H}_{c}$. A widely-used material is a cheap grain-oriented FeSi, and for precise applications 50\% $\mathrm{Ni}-\mathrm{Fe}$ alloy is used. By proper annealing, the coercivity of $50 \% \mathrm{Ni}-\mathrm{Fe}$ can be decreased below $2 \mathrm{~A} / \mathrm{m}$. It is also important to mount the core properly to avoid temperature-induced stresses. Widely-used epoxy coatings degrade the performance at temperatures below $0{ }^{\circ} \mathrm{C}$ (Fig. 13). The non-homogeneity of magnetic materials has an influence on the linearity of open-loop current sensors. By increasing the airgap, the influence of this nonlinearity is decreased, as is the influence of the remanence. The hysteresis error is approximately 0.2 to $0.5 \%$ for a $1 \mathrm{~mm}$ airgap and different grades of $50 \% \mathrm{Ni}-\mathrm{Fe}$ [Waeckerle 2006]. However, a larger airgap leads to unwanted sensitivity to the position of the measured conductor within the core, and also reduces the suppression of external currents and fields.

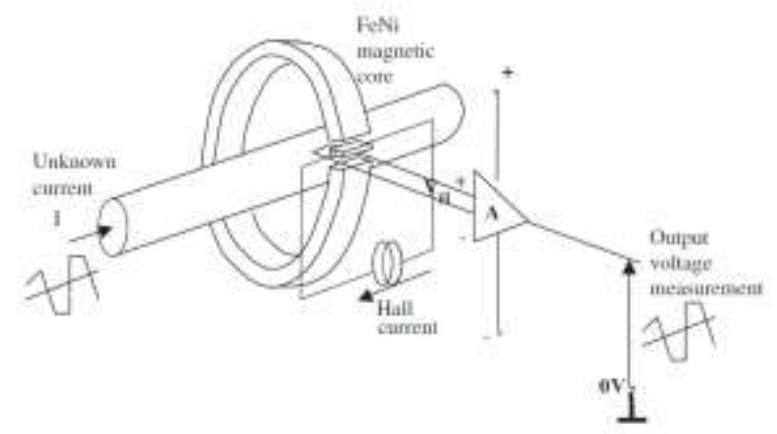

Fig. 12 A Hall DC current sensor in open-loop configuration - from [Waeckerle 2006], with permission from Elsevier.

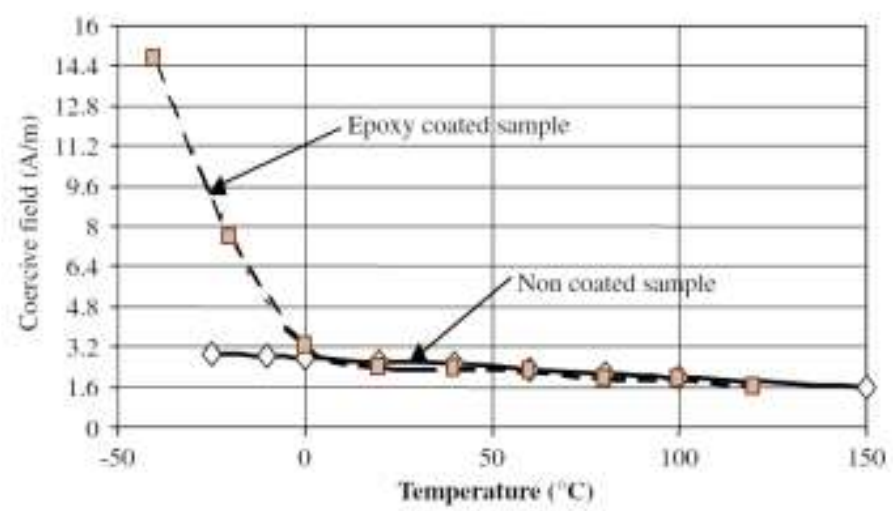

Fig. 13 Coercive field variation with temperature of Supranhyster50 in the coated and uncoated states - from [Waeckerle 2006], with permission from Elsevier.

The sensor linearity can be increased by using the feedback principle. Feedbackcompensated devices cancel the field in the yoke by using multiturn compensation winding. These sensors can achieve $0.02 \%$ error and a temperature coefficient of sensitivity of only $50 \mathrm{ppm} / \mathrm{K}$. However, the main weak point is still the limited zero stability due to the Hall sensor offset: the typical offset drift of a 50 A sensor is $600 \mathrm{~mA}$ in the $\left(0^{\circ} \mathrm{C} \ldots 70^{\circ} \mathrm{C}\right)$ range. This parameter is 20 times worse than that of fluxgate-type current sensor modules. 
Some Hall current sensors use field concentrators, which increase the measured field but do not completely surround the measured current. In this case the danger of saturation is much lower, but the position of the measured conductor should be fixed, and suppression of the external fields and currents should be achieved by other means. A low-cost current sensor based on a highly sensitive Hall sensor with simple integrated flux concentrators is described in [Popovic 2006] (Fig. 14). The field concentrators transform a lateral field locally into the vertical direction (Fig. 15). The sensor is manufactured by sentron (Melexis group) with $1 \%$ accuracy in the $\pm 12 \mathrm{~A}$ range. It can be used to measure the currents in both PCB conductors and free-standing conductors (Fig. 16).
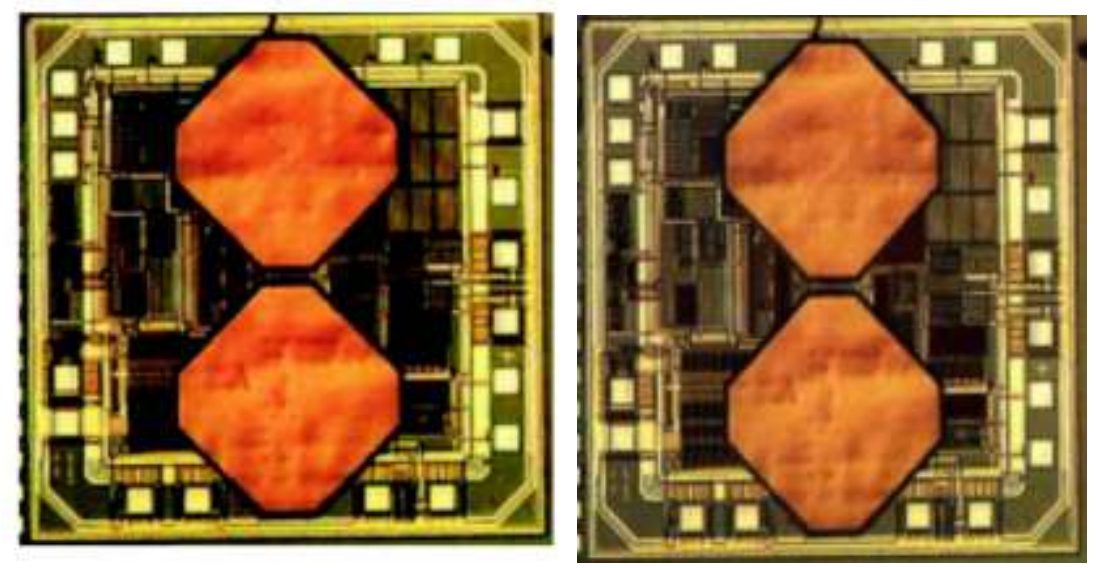

Fig. 14: internal structure of the CMOS integrated Hall magnetic sensor with twin integrated magnetic concentrators (Courtesy of Sentron AG)

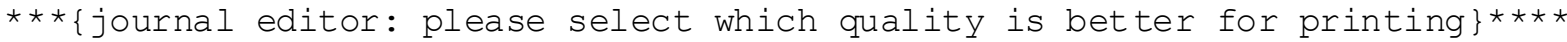

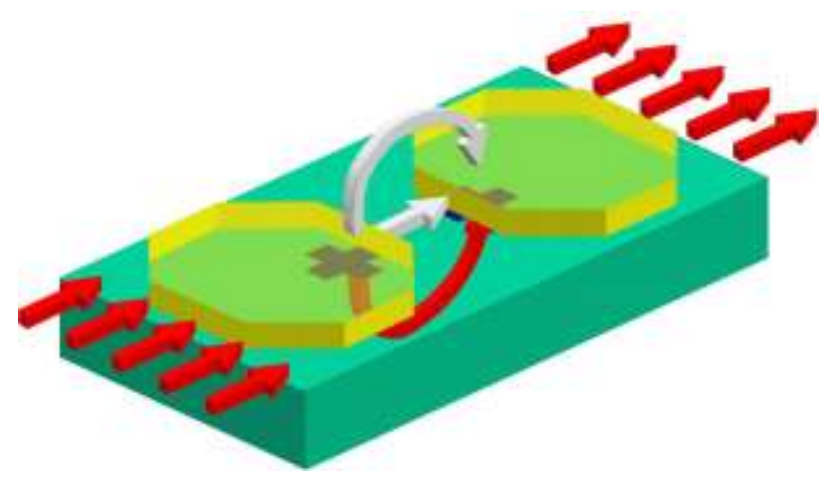

Fig. 15 Field concentrators deflect the flux into the vertical direction. The two small crosses under the concentrators are Hall sensors (Courtesy of Sentron AG).
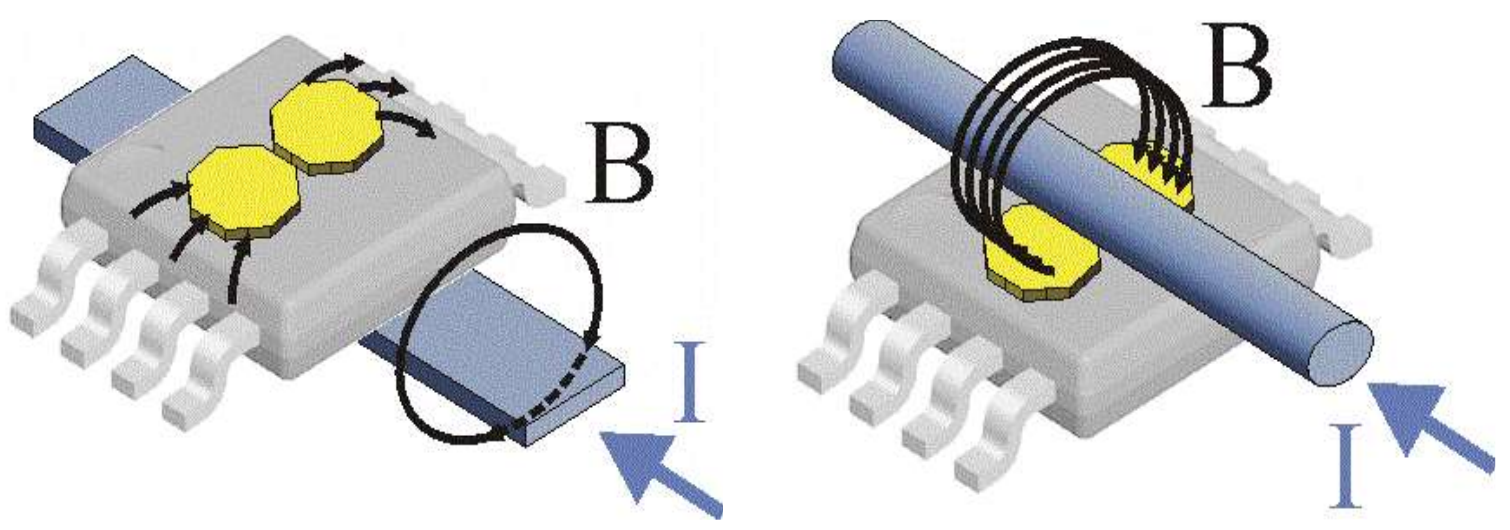

Fig.16: Current-sensing application of the CMOS integrated Hall magnetic sensor for a PCB conductor and a free-standing conductor. (Courtesy of Sentron AG) 
A yoke-less current transducer with six Hall-probes around the rectangular bus bar achieved $0.5 \%$ linearity and 0.2\% temperature stability in the $100 \mathrm{kA}$ range [Scoville and Petersen, 1991]. The effect of a $100 \mathrm{kA}$ return current at $50 \mathrm{~cm}$ distance was suppressed by a factor of 100 . Instead of simple averaging of the sensor outputs, crosstalk error can be more efficiently suppressed by the algorithm suggested by DiRienzo [2001].

An integrated current sensor based on two Hall sensors placed on both sides of the current strip was developed by Frick [2007]. The differentially connected Hall sensors reject the common-mode inference from the external magnetic fields (Fig.17). Proper autobalancing is achieved using built-in calibration coils. The achieved accuracy was $0.5 \%$ in the $5 \mathrm{~A}$ range with $1.5 \mathrm{kHz}$ bandwidth.

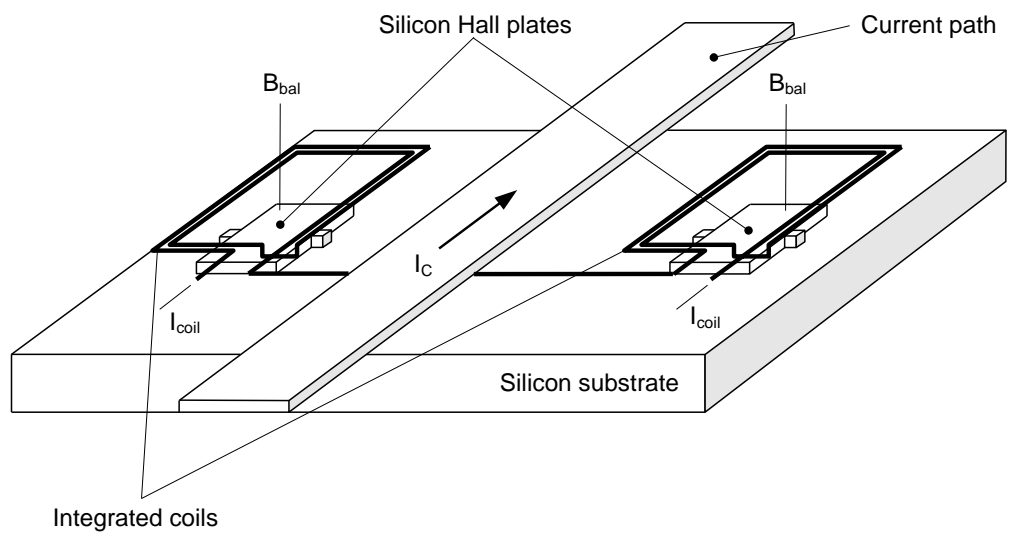

Fig. 17 A differential Hall current sensor with integrated calibration coils, after Frick [2007], reprinted by permission of Taylor \& Francis

Six Hall sensors can be used to measure currents in a three-phase conductor system. The achieved accuracy was $0.5 \%$ of the rated current and 4.5\% for the 10times overcurrent in a 0-20 kA amplitude range and in the 50-500 Hz frequency range. These are parameters sufficient for the requirements of an industrial "5P" current transformer [D'Antona 2001].

Some current sensors use a combination of a Hall sensor (for the DC and lowfrequency component) and a current transformer (for the high-frequency component), using a gapped ferrite core. A frequency range of $30 \mathrm{MHz}$ was achieved for 40 A range [Dalessandro 2007]. The matching between the two sensors can be made without an electronic stage. The idea is as follows: a high-frequency current transformer should have a low number of turns, which increases the lower corner frequency. The Hall sensors are therefore added to cover this frequency region.

The background calibration using integrated coils together with the spinning current technique can reduce the temperature sensitivity drift of the Hall sensor to less than $50 \mathrm{ppm} /{ }^{\circ} \mathrm{C}$, while the bandwidth can be as high as $500 \mathrm{kHz}$. Sensitivity variations due to mechanical stresses and ageing are also compensated [Pastre 2007]. This technique can significantly improve the performance of current sensors, even if feedback compensation is not possible.

Magnetic shielding can be used to isolate the coreless current Hall sensors from the influence of the non-measured currents. The difference between yoke and field concentrators, on the one hand, and shielding, on the other, is that, in the case of shielding, the sensor is not in the airgap of the magnetic circuit. An example of such a system is a three-phase 400 A current sensor for a power converter. The thickness of the U-shaped shields should be $1.5 \mathrm{~mm}$ to avoid saturation and to suppress the neighbouring currents by a factor of 100 [Kim 2009].

\subsection{XMR current sensors}

XMR is a common short form for magnetoresistors: anisotropic (AMR), giant (GMR), tunneling (TMR), colossal (CMR) and all other MRs. AMR sensors employ Anisotropic 
MagnetoResistance. They have higher resolution than Hall sensors, but their sensing direction is in the chip plane, so they cannot be built into the narrow airgap in the magnetic yoke. Yokes with a large airgap suffer from field leakage sensitivity to external currents and magnetic fields. Thus most current sensors based on AMR magnetoresistors are yokeless. The popular configuration is again a bridge measuring the magnetic gradient from the current, since it is resistant against homogeneous external magnetic fields (e.g. from distant currents). The bridge also suppresses the temperature variation of the electric resistivity of the magnetoresistive material (Fig. 18). This type of sensor usually has the current conductor integrated with the sensors in a single device to ensure stable geometry. The bridge has all the barber poles in the same direction, which means it is made insensitive to a homogeneous external field, but sensitive to measured current through the bus bar [Lemme and Friedrich, 2000]. The measured current can be compensated by a feedback current through a compensation conductor. A typical application is galvanically isolated current sensing in a PWM (pulse width modulation) regulated brushless motor. These sensors are manufactured by Sensitec (also under the F.W. Bell label) with ranges from 5 to 220 A. The achieved linearity is $0.1 \%$, temperature coefficient of sensitivity is $100 \mathrm{ppm} / \mathrm{K}$, and the offset drift in the $\left(-45^{\circ} \mathrm{C}\right.$ to $\left.+85^{\circ} \mathrm{C}\right)$ range is $1.4 \% \mathrm{FS}$ [Sensitec].

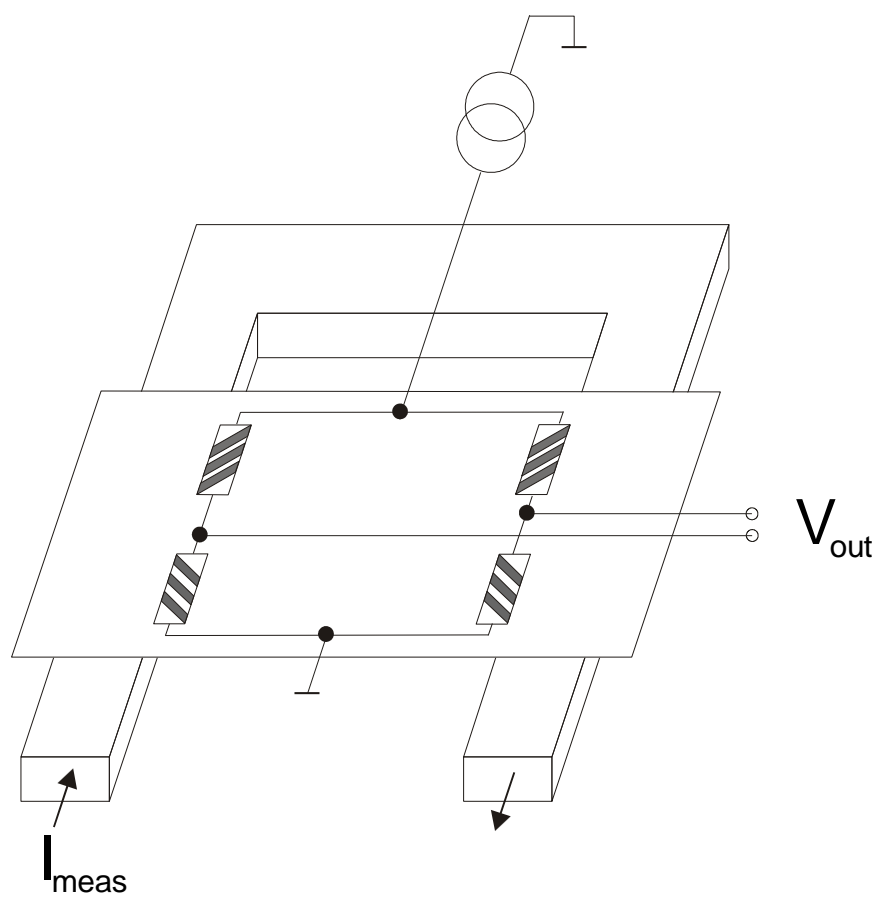

Fig. 18 AMR current sensor - after [Ripka 2004]

A similar sensor with a GMR detector (which employs Giant MagnetoResistance) has been developed by Siemens [Vieth 2000] and later improved by Reig [2004], and Pannetier-Lecouer [2007]. While the linearization of AMR sensors is achieved by using barber poles, GMR sensor technology does not have such a "geometric trick". To make the GMR bridge sensitive to the magnetic fields created by an electric current, various techniques can be used:

1. use sensors with two opposite response characteristics: in spin-valve GMRs this can be done by using hard magnetic layers with two opposite directions. Vieth [2000] used two separate sensor chips. It is also possible to manufacture such a structure on a single chip using two-step deposition with reversed direction of the magnetic field.

2. use a meander shape of the current path in order to reverse the field direction for two of the magnetoresistors. An FEM (finite element) analysis of such a sensor was made by Beltran [2007]. The advantage of this method is that 
the complete bridge with four active GMR sensing elements can be made simultaneously on a single chip, so that their properties are identical and the temperature compensation is very effective Reig [2004]. A similar approach was used by Pannetier-Lecouer [2007].

3. inactivate two of the magnetoresistors, e.g. by shielding

Fig 19 shows the spin-valve current sensor using a meander current path. The four GMR strips $R_{1}-R_{4}$ appear as short horizontal lines. They are exactly the same, with the pinned layer easy axis and free layer easy axis denoted by solid arrows. The measured current flows from left-to-right below $R_{1}$ and $R_{3}$, and from right-toleft below $R_{2}$ and $R_{4}$. Therefore the associated magnetic field is parallel to the pinned layer easy axis of $R_{2}$ and $R_{4}$, and antiparallel to the pinned layer easy axis of $R_{1}$ and $R_{3}$. Then the magnetic field forces the free layer magnetization vector, initially perpendicular to the magnetic field, to rotate (see Fig.

$19(\mathrm{a}))$. As a result, for a given current direction, $\mathrm{R}_{1}$ and $\mathrm{R}_{3}$ increase and $\mathrm{R}_{2}$ and $\mathrm{R}_{4}$ decrease. Fig. 19 (b) shows how the resulting Wheatstone bridge works. 

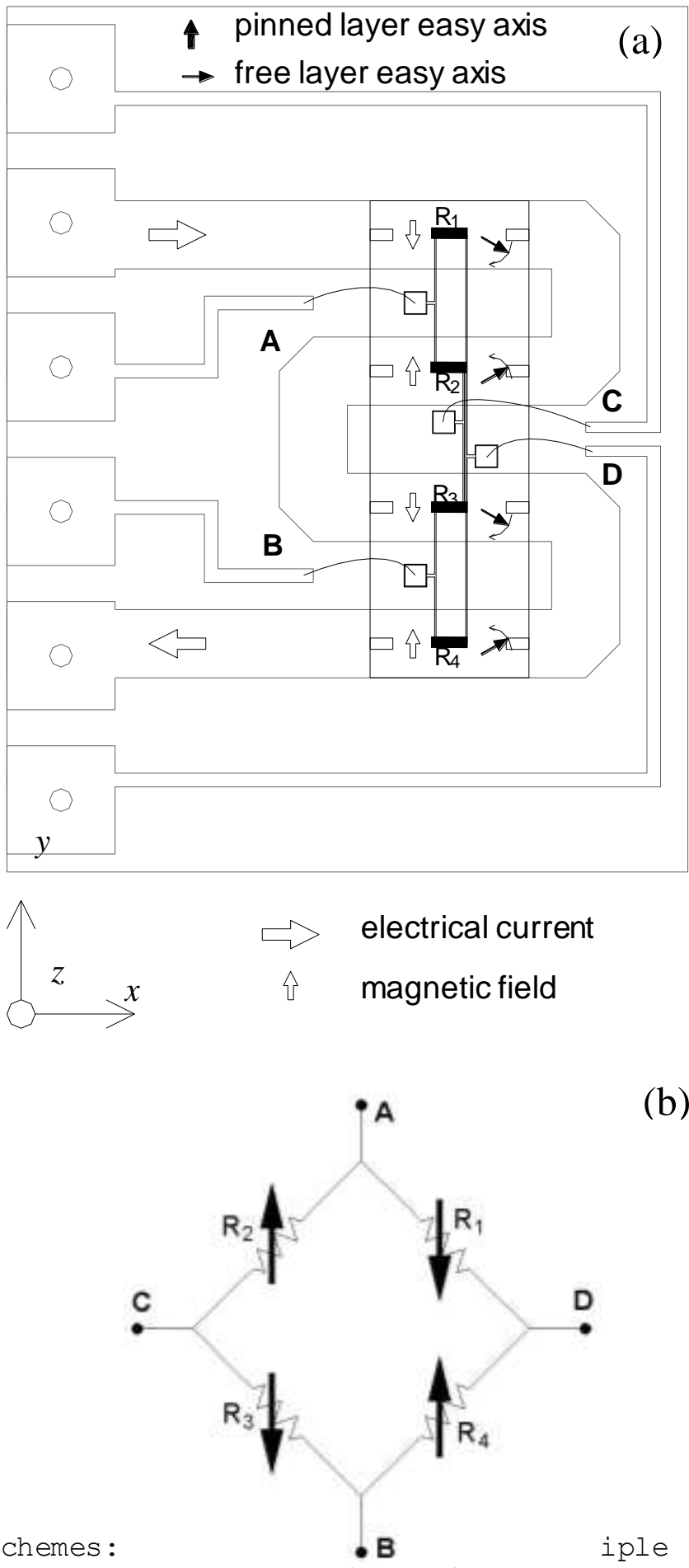

(b)

Fig. 19 Sensor schemes:

- B

iple (notice that due to the scale the conductor traces are not discernible);

(b) Wheats tone

bridge equivalent - from [Reig 2004], with permission from Elsevier.

In some of these sensors, additional biasing was achieved by permanent magnets in order to increase the linearity and stabilize the sensors against large external fields. Proper techniques such as feedback compensation should be used to compensate for the temperature dependence of the biasing magnet.

GMR sensors may exhibit a large irreversible change of characteristic after a magnetic shock created by a large external field or overcurrent.

Another possible configuration is a multi-sensor arrangement in a circular pattern around the measured conductor. This can be used for any magnetic sensor - 
especially Hall sensors - to measure large currents, and for AMR sensors to measure lower currents.

If we only make a summation of the sensor outputs, the error caused by displacing the measured current from the center position depends strongly on the number of sensors. Fig. 20 shows the simulated results: with 4 sensors the maximum theoretical error is 3\%, while for 8 sensors it is below $0.1 \%$.

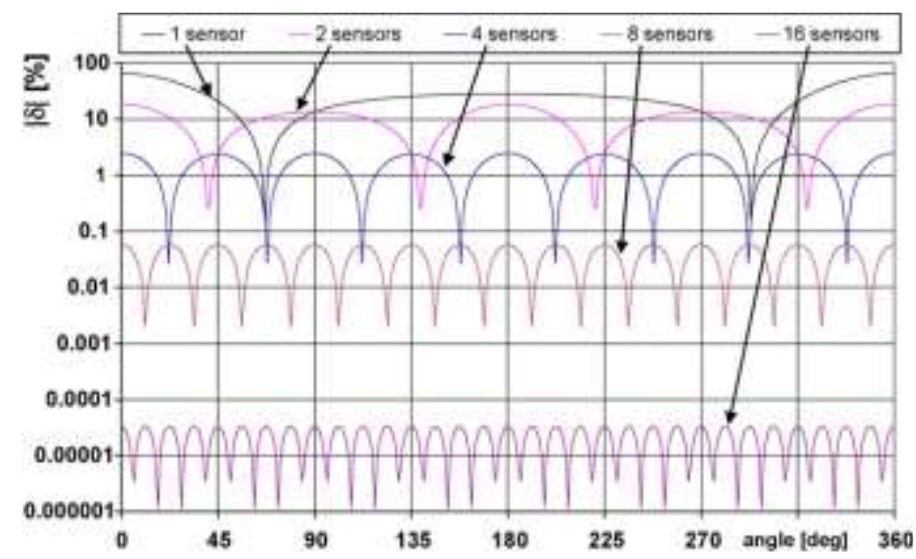

Fig. 20 The theoretical dependence of the relative error of the current determination, when the wire rotates around the measurement hole for various numbers of sensors. Diameter of the hole $10 \mathrm{~mm}$, diameter of the sensor array $25.4 \mathrm{~mm}$ (1 in.) - from [Mlejnek 2008], reproduced with permission from Elsevier

A linearity error of $\pm 0.05 \%$ in the current range of $\pm 8 \mathrm{~A}$ was achieved using 8 Philips KMZ51 AMR sensors (Fig. 21). The error caused by an uncentered wire is $\pm 0.5 \%$, which is 5-times worse than predicted by the numerical simulation. This degradation is caused by differences between the sensitivities of the individual uncompensated sensors. An external current of $5 \mathrm{~A}$ at a distance of $40 \mathrm{~mm}$ from the sensor array center causes an additional absolute error of max. $25 \mathrm{~mA}$. [Mlejnek $2008]$.

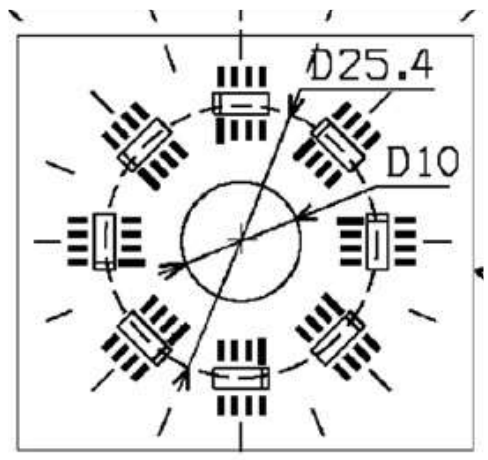

(a)

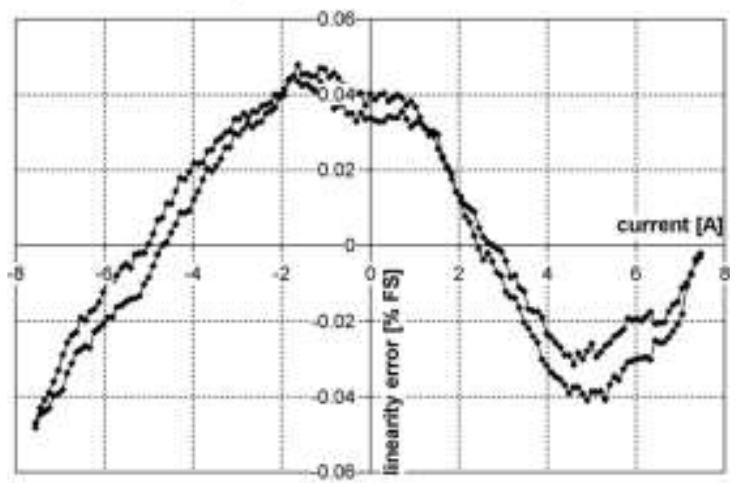

(b)

Fig. 21 a) The AMR current probe layout, b) the linearity error for a centered wire and a compensated sensor-from [Mlejnek 2008], reproduced with permission from Elsevier 


\subsection{Magnetooptical current sensors (including fibre-optic)}

Optical current sensors have several advantages which are very attractive for power distribution applications:

1. Effective isolation from high potentials

2. Immunity against electromagnetic interferences

3. High dynamic range, no saturation effects

4. High bandwidth

5. Compact and lightweight design.

These features offer a significant cost reduction in comparison to conventional high-voltage current transformers.

Most optical current sensors are based on the Faraday Effect - either in bulk material or in an optical fibre.

The polarization plane of a linearly polarized light which travels through the magneto optical material is rotated by angle $\alpha$, which is given as

$$
\alpha=V \oint_{C} B \cdot d l
$$

where $\mathrm{V}$ is the Verdet constant, $\mathrm{B}$ is the magnetic field strength dl is the line element along the optical path inside the material.

Magneto optical current sensors are ideally suited for high-voltage high-current applications [Cruden 1998].

\section{Detection schemes}

Most of these devices use either the interferometric principle or the polarometric principle. The interferometric configurations utilize a Sagnac interferometer, which we will discuss later in the section on intrinsic opticalfibre sensors.

The basic polarometric detection scheme consists of a polarizer at the sensor's input to generate a linearly polarized optical state, and an analyser at the output which converts the polarization change due to the Faraday Effect into an intensity measurement. In common applications, the cross-polarization angle between the two polarizers is set at $45^{\circ}$. However, a different angle may bring higher immunity to the noise caused by the sensitivity of the interconnecting optical fibres to mechanical vibrations [Fisher 1995]. It has been shown that the major source of the noise is birefringence induced by the external vibrations acting on the up-link fibre. Another noise-rejection scheme utilizes two downlink optical fibre leads: one carries the signal before the analyzer (noise only), while the other carries the signal after the analyzer (Faraday signal + noise). Noise rejection is performed by subtracting the intensity signal from these two downlink fibres [Fisher 1996a].

Some sensors use a dual-frequency or polychromatic light source and chromatic sensing. This utilizes the wavelength dependency of the Verdet constant V. The use of two or more photo detectors may effectively compensate for temperature dependencies and other stray effects.

\section{Bulk Magneto optical sensors}

Magneto optical point sensors (or unlinked sensors) use a piece of glass or a crystal rod placed in the neighbourhood of the electrical conductor. The sensor is usually interrogated by optical fibres. These devices are robust, cheap, and sensitive. They belong to the class of "extrinsic fibre sensors", i.e. sensors which use optical fibres for transmission, not for sensing. These sensors are employed in the first generation of the ABB magneto-optic current transducer (MOCT), which served for more than 15 years in industrial applications. The ABB sensor achieves an accuracy class of 0.2 in the 3000 A range. 
Using a bulk flint glass optical detector in the $20 \mathrm{~mm}$ wide airgap of a ferromagnetic yoke, a noise level of $1.6 \mathrm{~mA} / \sqrt{\mathrm{H}} \mathrm{z} 280 \mathrm{~Hz}$ was achieved. However such a large airgap should significantly reduce the geometrical selectivity [Yi 2002]. Yoshino [2001] used a transverse configuration of the light beam and currentinduced magnetic field. This sensor requires only a $3 \mathrm{~mm}$ airgap so that the surrounding currents are better suppressed. In any case, using a ferromagnetic yoke brings the danger of saturation.

The triangular prism bulk magneto optical sensor has a closed sensing optical path around the measured conductor. This provides independence of the sensor output from the position of the measured conductor within the closed path, and also resistance to external conductors and external homogeneous magnetic fields. The sensor configuration is shown in Fig. 22. The noise rejection scheme using two down links is also shown here [Fisher 1996b].

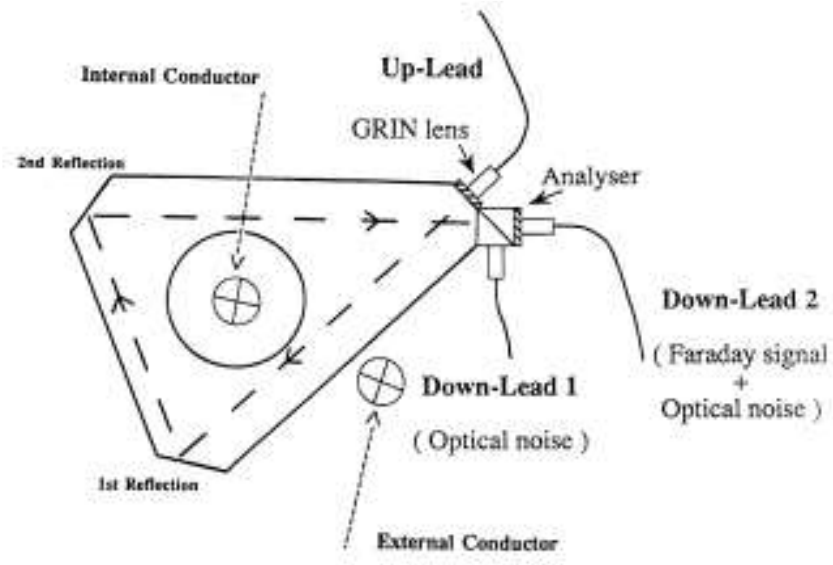

Fig. 22 Bulk-optic triangular Faraday current sensor. The position of the internal (measured) conductor and external conductor is also shown - from [Fisher 1996a].

In order to increase the sensitivity, light can be passed several times around the conductor using multiple reflections. A sensor with three 3-D loops using total reflection at the glass surface is shown in [Ning 1995]. If total reflection is used, the optical attenuation is low, but multiple reflections cause elliptical polarization [Li 1999].

When measuring a very large current, the sensor information can be ambiguous, as the phase shift may exceed $360^{\circ}$. This can be solved by counting how many times the phase crossed $0^{\circ}$. This technique was used to measure $720 \mathrm{kA}$ current pulses using a $100 \mathrm{~mm}$ x $100 \mathrm{~mm}$ Schott glass (SF4) sensing element excited by a $532 \mathrm{~nm}$, $100 \mathrm{~mW}$ solid-state laser [Deng 2008].

Broad-band light sources such as LEDs are often used in these devices. The dominant source of dispersion is the wavelength dependence of the Verdet constant. It was theoretically proven that that the error accumulation due to spectral width variation is so small that it can be neglected, and a monochromatic model can be used even for broadband optical current sensors [Wang 2005 ].

If bulk magneto optical sensors are used to measure the current in three-phase systems, compensation should be made for the crosstalk from other conductors [Perciante 2008].

Faraday mirrors can also be used to measure current: these sensors are also called orthoconjugate reflector (OCR) current sensors. While the birefringence in the glass current-sensing head causes the plane of polarization to rotate by approximately $20^{\circ}$, Faraday mirror sensors exhibit only $5^{\circ}$ birefringence [Wang 2007]. 


\section{All-fibre sensors (or intrinsic fibre sensors)}

In wound fibre devices, too, the magneto optical material encloses the electrical conductor, and thus these sensors are not sensitive to external currents and magnetic fields.

Optical fibre is made from materials that have much lower Verdet constants than the magneto optic crystals used for bulk sensors, but their sensitivity can be increased by using a higher number of turns of the fibre wound around the measured conductor. Fibre-optic sensors are simple devices, they suffer from the spurious birefringence induced in bent fibres [Perciante 2008].

Sensors with back light propagation can be constructed to compensate for birefringence. This approach exploits the non-reciprocity of the Faraday Effect and the reciprocity of linear birefringence. The light wave is reflected on the far end and its polarization state is rotated by $90^{\circ}$. Then, it is coupled back into the fibre [Drexler 2008]. Asensor of this type, made of low-birefringent flint fibre with a very low photo elastic constant, achieved the accuracy required for the $0.1 \%$ class of current transformers in the range of $1 \mathrm{kA}$ [Kurosawa 2000].

All-fibre sensors can be made flexible using back-and-forth propagation through a twisted sensing fibre [Alasia 2004]. Such sensors can be wound around the measured conductor on existing installations.

A polarimetric current sensor utilizing a fibre-laser was reported in [Lee 1998]. The output of this sensor is a frequency, and it is immune to intensity perturbations. $1 \mathrm{~mA}$ resolution for AC current was achieved for 1 turn of the measuring fibre. Although the results are promising, this scheme has not been applied in industry due to its complexity.

Sagnac interferometer-type fibre optic sensors have the big advantage that they can use the technology originally developed for fibre-optic gyros [Takahashi 2004]. The early models used polarization maintaining (PM) fibres, which are expensive and difficult to install. Even with the best PM fibres, polarization phase noise was still a problem. Figure 23 shows the configuration, using a depolarizer and a single-mode (SM) sensing fibre. The light source is a $0.85 \mu m$ super-luminescent diode (SLD). The light passing through the sensing fibre coil is circularly polarized by fibre polarizers and 1/4-wavelength plates at both ends of the sensor fibre. The detection scheme utilized a PZT piezoelectric modulator and complicated demodulation. The rated current of the sensor designed by Takahashi [2004] is 3000 A, and the maximum measured current is $100 \mathrm{kA}$. Such a large dynamic range allows the use of the same device for measurement and protection purposes. The birefringence of the sensing fibre was suppressed using a twisted double-coated low birefringence fibre. The sensor linearity was better than 0.2\%. Figs. 24 and 25 show the ratio and phase errors as a function of the measured current. The achieved errors are very small, and they do not

significantly increase at low currents. In the temperature range of -40 to $+60^{\circ} \mathrm{C}$, the maximum ratio error was $\pm 0.2 \%$ (Fig. 26). All these characteristics apply to AC $50 \mathrm{~Hz}$ or $60 \mathrm{~Hz}$ currents. When measuring DC currents, this sensor suffers from $10 \mathrm{~A} / \mathrm{h}$ drift caused by $0.05 \mathrm{mrad}$ non-reciprocal phase shift. The temperature dependence of the Verdet constant of the sensing fibre $\left(0.69 \times 10^{-4} \mathrm{~K}^{-1}\right)$ is compensated. 


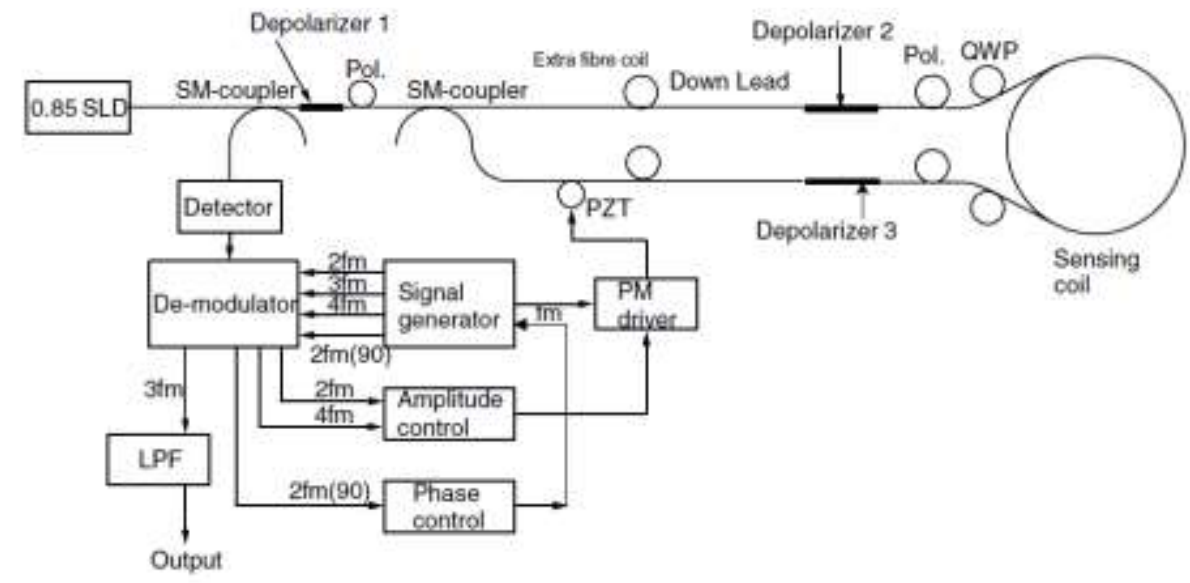

Fig. 23 Schematic diagram of the fibre-optic current sensor -from [Takahashi $2004]$.

Fig. 24 Ratio error characteristics of the fibre-optic current sensor - from [Takahashi 2004].

Fig. 25 Phase displacement characteristics of the fibre-optic current sensor-

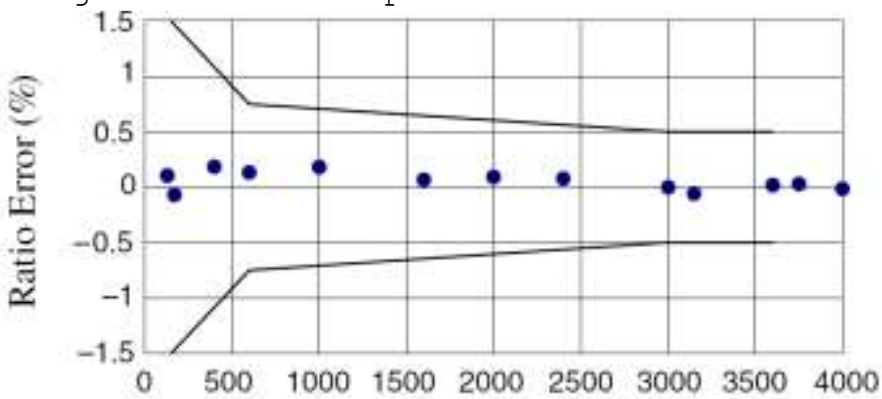

Primary current ( $\left.\mathrm{A}_{\mathrm{RMS}}\right)$

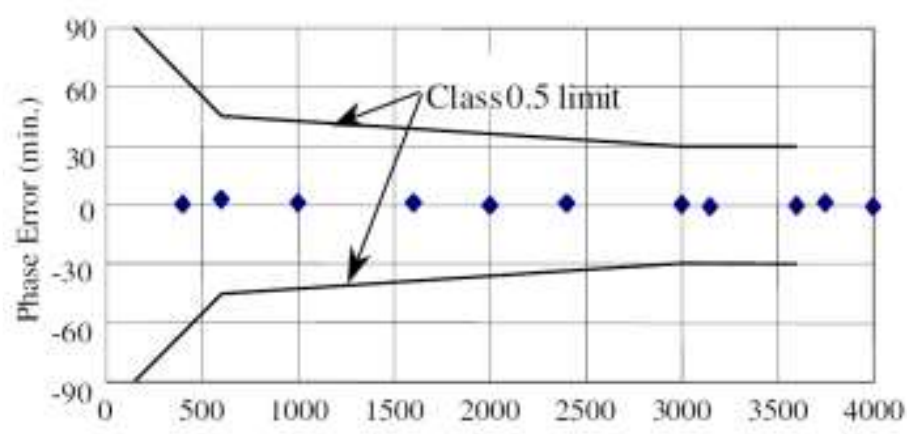

Primary current ( $\left.\mathrm{A}_{\mathrm{RMS}}\right)$

from [Takahashi 2004]. 


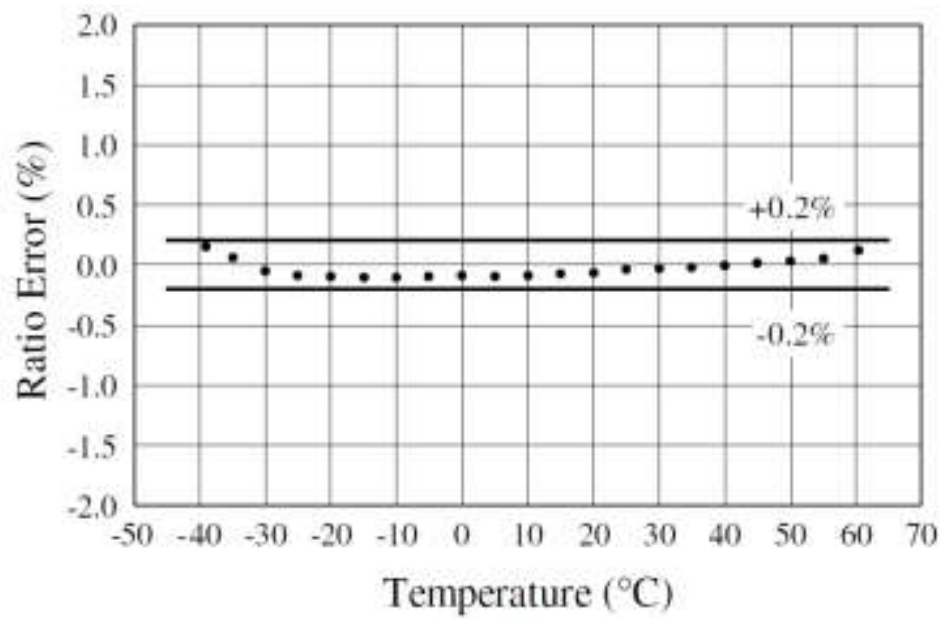

Fig. 26 Temperature characteristics of the fibre-optic current sensor - from [Takahashi 2004].

A commercially available all-fibre current sensor was manufactured by ABB [2005] . This device also uses two circularly polarized light waves, but travelling in the same direction. Two linearly polarized light waves with orthogonal polarization are passed through the optical fibre. At the point where the sensitive region starts, the waves are converted by the phase retarder into left and right circularly polarized light waves. The magnetic field caused by the measured current causes a nonreciprocal phase shift between the two beams by the Faraday Effect. Both waves are reflected at the end of the fibre with swapped polarization, and return back to the optical module. On leaving the measuring coil, the circular waves are converted back to linearly polarized light. This configuration effectively suppresses the bending-induced linear birefringence in the fibre, which can be in the order of $5^{\circ}-10^{\circ}$ per coil turn, with the orientation within the coil plane [Zhou 2007]. The phase difference caused by the magnetic field is then measured by the interferometer. The measuring range is 600 $\mathrm{kA}$, corresponding to $360 \mathrm{deg}$. phase difference, while the resolution is $0.25 \mathrm{~A}$. The sensitivity can certainly be increased by using multiple turns of the optical fibre around the measured conductor. Great care was taken to insulate the measuring fibre from mechanical stress and vibrations, as this is a major source of temperature sensitivity drift. The sensing fibre is thermally annealed to remove residual stresses and covered by a glass capillary, which is embedded in a soft polymer ring.

The temperature dependence of the Faraday Effect $\left(0.7 \times 10^{-4} /{ }^{\circ} \mathrm{C}\right)$ is partly compensated by the temperature dependence of the retarder.

Similar sensors were developed for measuring large DC currents: the achieved accuracy is $0.1 \%$ for currents up to $600 \mathrm{kA}$ [Bohnert 2007].

Fibre optic current sensors allow multiplexing of other signals using the same fibre. A system combining a polarometric current sensor based on the Faraday Effect with a voltage sensor based on the Pockels effect is described in [Ferrari 2009]. The Faraday sensor works in the violet region, where the Verdet constant is large, while the Pockels cell works in the green region. A third beam, which is blue, serves as a reference. This approach is limited by the low accuracy of the Pockels cell and by the non-ideal characteristics of the filters.

\section{Other types of extrinsic fibre optic current sensors}

These sensors utilize optical fibres for connection to a separate sensing element. The first class of these sensors comprises bulk magneto optical sensors (Section 4.8.1). Other current sensors can also be used; the required electric supply energy can be converted from the incoming light. However, using active components at high potential is dangerous, as they can easily be destroyed in a strong electric field during voltage transients. Completely passive sensors can be built using the secondary current of the instrument current transformer to directly excite the light-emitting diode (LED], which sends light through the downlink optical fibre. Using the wavelength shift of green ultra-bright LEDs instead of intensity modulation can overcome some of the problems with drifts and 
noise, but the LED temperature stability is still a problem [Ribeiro 2008]. A current clamp transformer was used to directly excite the piezoelectric PZT element attached to Fibre Bragg grating (FBG) [Fisher 1997]. The measured DC or AC current is converted to the strain of the FBG, resulting in a wavelength shift. The FBG is illuminated by a remote broadband source, and the resonance frequencies of all 3 FBGs working at different frequencies are measured using an all-fibre Mach-Zehnder interferometer [Jackson 2009].

Fibre optic cables are also used in magneto-strictive current sensors (Section 4.7).

\subsection{Other principles for current sensing}

\section{A GMI current sensor}

A Giant MagnetoImpedance (GMI) current sensor was reported in [Malatek 2005]. An amorphous $\mathrm{CO}_{67} \mathrm{Fe}_{4} \mathrm{Cr}_{7} \mathrm{Si}_{8} \mathrm{~B}_{14}$ strip was annealed to have $230 \%$ GMI at 20 MHz. The strip was wound around the measured conductor and was DC biased by an external coil to achieve a linear response. A double-core structure was used in order to improve the temperature stability. Further utilization of AC biasing (up to $200 \mathrm{~Hz}$ ) of the double-core sensor suppressed the temperature offset drift by a factor of 30 (down to $0.6 \mathrm{~mA} / \mathrm{K}$ ) and increased the open-loop linearity to $0.5 \%$ for the $2-\mathrm{A}$ range.

The GMI current sensor reported by Z. Zhan [2007] has only a single core, but the reported zero stability is $0.25 \mathrm{~mA} / \mathrm{K}$ in the $+/-2.5 \mathrm{~A}$ range. The accuracy is 0.45 ․

An asymmetric GMI was also used for current sensing. The current sensitivity for a single-strip sensor is $0.13 \mathrm{~V} / \mathrm{A}$ at $100 \mathrm{kHz}$ operating frequency, and increases to $0.94 \mathrm{~V} / \mathrm{A}$ at $1 \mathrm{MHz}$. However the DC stability of these devices is expected to be low.

A double-core GMI sensor biased by permanent magnets is reported by Han [2009]. The measurement error is less than 0.16\% FS at room temperature. The claimed thermal stability is $0.01 \% \mathrm{FS} /{ }^{\circ} \mathrm{C}$ in the temperature range between -20 and $+30^{\circ} \mathrm{C}$. The main disadvantage of this sensor is the hysteresis error of $0.86 \%$ FS.

The temperature stability of GMI sensors was analyzed in [Malatek 2008]. The most serious source of temperature dependence is the temperature variation of the resistivity of the sensor material. Equivalent temperature offset drift -0.27 $\mathrm{A} / \mathrm{m} / \mathrm{K}(-340 \mathrm{nT} / \mathrm{K})$ is achieved only after using a special alloy with a very small negative temperature coefficient of resistivity. This parameter is better than for Hall sensors, but worse than for magnetoresistors and fluxgate sensors.

The papers mentioned above show that, despite intense development, GMI current sensors still do not match the parameters that are standard for well-established technologies.

\section{Superconducting current sensors}

SQUID (Superconducting Quantum Interference Detector) magneto encephalographs measure the fT fields produced by neural currents in the brain. SQUIDs can also be used to measure the small currents that pass through a coil inductively coupled to the SQUID loop. The SQUID should be shielded against external magnetic fields or made as a gradiometer with a very short baseline. SQUIDs normally measure only flux changes, but an array of SQUIDs can be used to build an absolute current sensor with 1 nA resolution [Beyer 2008]. As the SQUID is a nonlinear device (the voltage-flux characteristic is similar to a sinewave), the flux should be compensated by a flux-locked loop. This is a technical problem at higher frequencies. A noise level of $7.4 \mathrm{pA} / \sqrt{ } \mathrm{Hz}$ up to $10 \mathrm{MHz}$ was achieved using a two-stage SQUID instead of an external op-amp [Drung 2009].

An ion beam current monitor with a high-temperature superconducting (HTS) SQUID has been developed by Watanabe [2008] for application in the RIKEN cyclotron. A schematic drawing of the improved bridge circuit is shown in Fig. 27. While a beam passes through the HTS superconducting tube along its axis, a shielding current produced by the Meissner effect flows in the opposite direction along the wall, so as to screen the magnetic field generated by the beam. The whole circuit 
is completely surrounded by high-permeability materials to shield it from external magnetic fields. A high-permeability core is used to increase the flux created by the measured current by a factor of 50 (Fig. 27 c,d,e). The relative permeability of this $80 \% \mathrm{Ni}$ core is 4000 at room temperature and 2500 at the temperature of liquid nitrogen. The resulting resolution is $10 \mathrm{nA}$.

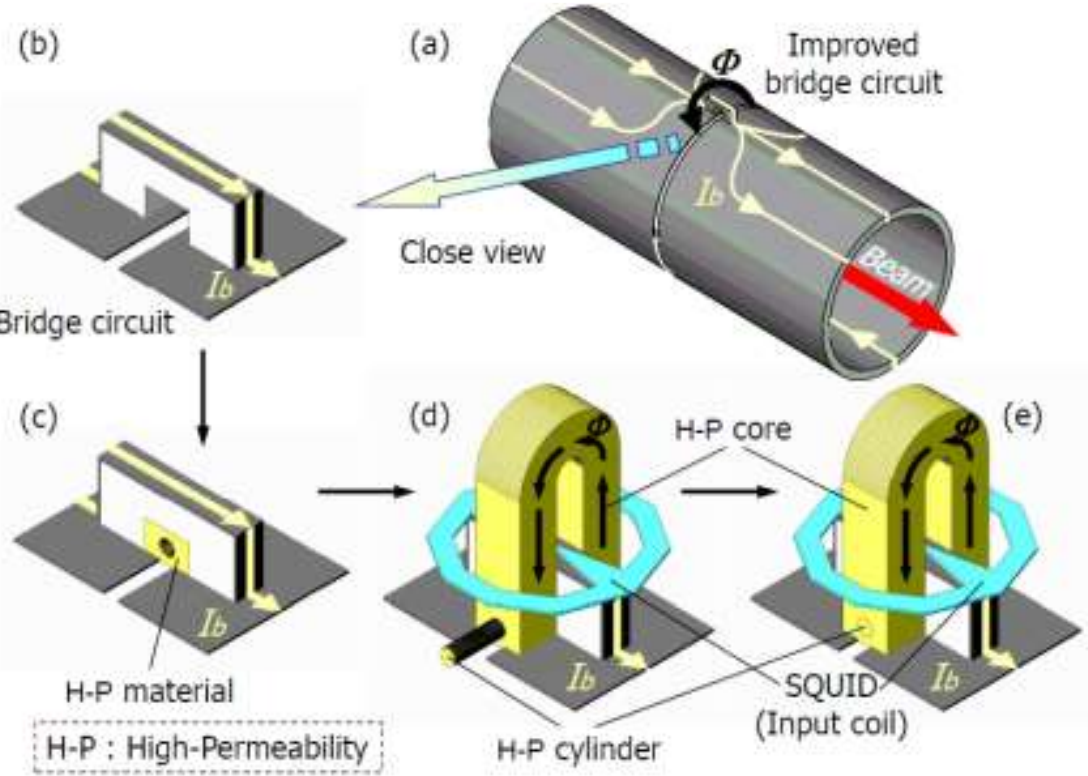

Fig. 27 SQUID current monitor for Ion beam - from Watanabe [2008], with permission of IOP.

A current amplifier operating at $4.2 \mathrm{~K}$ is described in [Gallo 2000]. A cryogenic current comparator with a current ratio of 10 000/1 and low noise dc SQUID magnetic shielding are combined to achieve an equivalent input noise of $4 \mathrm{fA} / \sqrt{ } \mathrm{Hz}$ for frequencies higher than the flicker noise corner of around $0.5 \mathrm{~Hz}$.

\section{Magnetometric location and measurement of hidden currents}

The field from a long straight conductor decreases with 1/r, assuming that the return conductor is at a large distance. If this is not the case, the magnetic field is lower, and it should be calculated from the actual geometry. The other extreme is a small current loop, which creates a field decreasing with $1 / r^{3}$ distance. Underground electric conductors can be located and their current can be remotely monitored by measuring their magnetic field at several points. In the case that the cable contains both forward and return currents, it can be detected only from a small distance, and the current value cannot be measured. This technique was used for locating underwater optical cables which contain a metallic conductor delivering a DC current of about 1 A to supply the repeaters. The field distribution was measured by two three-axial fluxgate magnetometers. The cables were detected from a distance of $40 \mathrm{~m}$, and their position was determined with $0.1 \mathrm{~m}$ accuracy from a distance of $4 \mathrm{~m}$ [Takagi 1996].

The magnetometric current is also used to locate and measure the AC and DC fault currents in building structures, such as bridges. The natural variation of the Earth's field induces currents in long conductors, which may cause electrochemical corrosion: a 70 A current was measured in the Alaska Oil Pipeline [Campbell 1980].

Sensitive magnetic sensors such as fluxgates should be used. It is an advantage if the sensor is vectorial. However, scalar sensors such as proton, electron-spin resonance (ESR) and atomic (optically pumped) magnetometers are also used to measure current. A current meter using ESR has been reported by Duret [1992]. ESR material with an extremely narrow resonance line is subjected to a polarization field and to a field proportional to the measured current. The ESR is detected at $40 \mathrm{MHz}$ for a polarization field of $1.4 \mathrm{mT}$. The extra field coming from the measured current creates a shift in the resonance line which is feedback compensated. The resolution is $10 \mu \mathrm{A} \sqrt{ } \mathrm{Hz}$. 


\section{Current sensors using MAGFETS}

Magnetically sensitive CMOS Split-Drain Transistors were used to measure current through the strip (using 126 transistors) or in a planar coil (using a single transistor). Both sensors were made in CMOS technology. The coil sensor has a noise of $2.8 \mu \mathrm{A} / \sqrt{ } \mathrm{Hz} @ 1 \mathrm{~Hz}$ and a full-scale range of $20 \mathrm{~mA}$, while the strip sensor has a noise of $42 \mu \mathrm{A} / \sqrt{ } \mathrm{Hz} Q 1 \mathrm{~Hz}$ and a range of $500 \mathrm{~mA}$ [Castaldo 2009]. These values are promising for future development; however, this device still does not match the parameters of similar Hall current sensors.

\section{Lorentz force sensors}

A sensor designed by Dinev [1996] is based on an elastic cantilever. The cantilever is made of aluminium-coated optical fibre. A conductor supplied with a measured current $I_{c}$ is placed parallel to the coated fibre (cantilever), at a distance $\mathrm{D}=5 \mathrm{~mm}$ (Fig. 28). Another current $I_{0}$ flows through the aluminum fibre jacket. The Lorentz force between the two currents causes deflection of the fibre, which is detected by a position-sensitive photodetector. The range of measured currents is $300 \mathrm{~mA}$ to $20 \mathrm{kA}$. The sensitivity is controlled by Io (Fig. 29). The critical disadvantage of this sensor is its sensitivity to external magnetic fields, which cannot be easily compensated. Other problems include sensitivity to temperature and gravity bending, and to vibration.

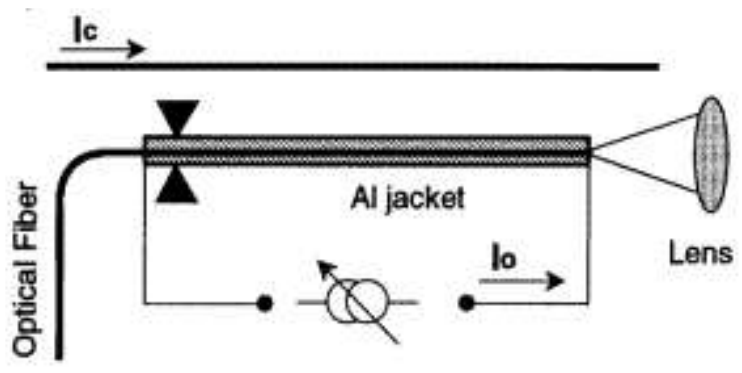

Fig. 28

Fibre-optic Lorenz force current sensor - from Dinev [1996]

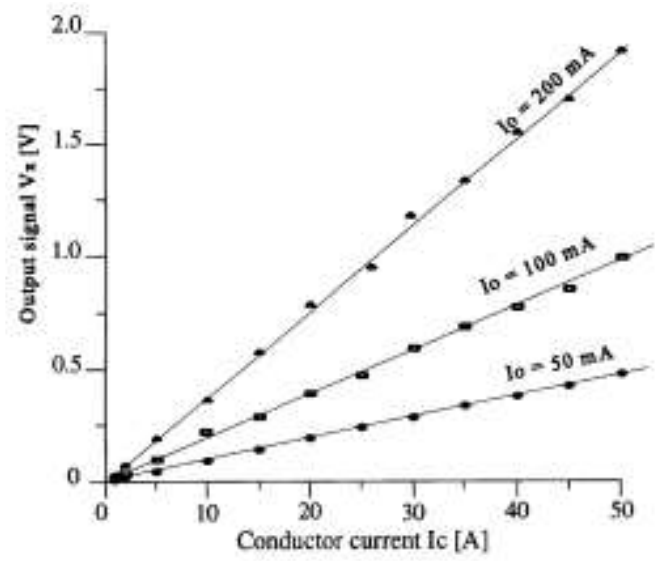

Fig. 29 The fibre-optic Lorenz force current sensor response calibration curve from Dinev [1996]

Magnetic force microscopy (MFM) allows remote measurement of currents in integrated circuits with high spatial resolution. The sensor tip is made of a magnetic material which is magnetized in the direction of the mechanical oscillation of the sensors. The magnetic field gradient generated by the measured current creates a magnetic force which is detected as a change in the amplitude or the phase of the oscillations. A conventional scanning force microscope is based on a vibrating cantilever. Using a quartz needle sensor, it is possible to inspect packaged devices through a window opened in an etching process. The needle oscillates at the mechanical resonant frequency with 1 nm amplitude at a distance of $1 \mathrm{~mm}$ from the surface. A large scanning height was selected in order 
to eliminate the influence of voltage caused by the potential difference between the probe tip and the conducting line. The detected current was 100 A [Hartmann 2005], with about $10 \mu \mathrm{m}$ spatial resolution.

\section{Magnetostrictive and force current sensors}

In the Fibre Bragg Grating current sensor, described by [Zhao 2006], [Reilly 2006] and [Cheng 2007], the optical fibre sensor measures the force on a magnetic element attracted by a solenoid, which is supplied by the measured current. The performance of these sensors is poor, and we consider their potential to be weak. Another approach is to combine a magneto-strictive material to create strain and a piezoelectric material to generate voltage from this strain, see e.g. [LopezGarcia 2006]. These devices, too, perform poorly in comparison with the previously described principles.

Some prototype current sensors are based on a force exerted on a small permanent magnet in the magnetic field gradient. The operation of such a "single-point" DC/AC magnetic gradient sensor with an optical readout is explained in [Lucas 2009]. This type of sensor can measure a current in a 2-wire appliance cord without the necessity to separate the two conductors physically. If we require only AC operation, the sensing element can also be a piezoelectric MEMS cantilever with a permanent magnet mounted on the cantilever's free end (Fig. 30). When placed near a wire carrying AC current, the magnet is driven sinusoidally, producing a voltage in the cantilever that is proportional to the current being measured [Leland 2009].

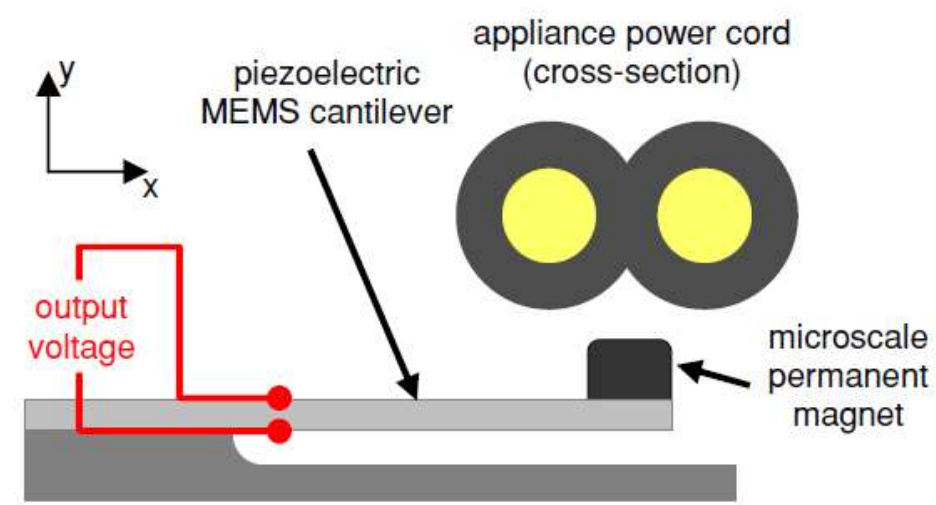

Fig. 30 Scheme of the MEMS current sensor design (not to scale). From [Lucas 2009], with the permission of IOP

Another ring-shaped magneto-strictive sensor consists of two Nizn magnetostrictive ferrite disk cores, a PZT piezoelectric disk sandwiched between them and a toroidally wound pickup coil. The piezoelectric disk is vibrated by an excitation voltage and, using the magneto-striction effect, it modulates the magnetic core flux associated with a measured current, which passes through the sensor's central hole. The time-varying flux induces an alternating voltage in the pickup coil. Multiple gaps in the ferrite rings reduce the hysteresis from $22 \%$ to 2.2\%, but the linearity error is still $0.8 \%$ in the 60 A range for a $9 \mathrm{~mm}$ inner diameter/ $16 \mathrm{~mm}$ outer diameter ring with 8 gaps [Koga 2009].

\section{Indirect sensing of inductor current}

Inductor current can be measured indirectly using its resistance if it is connected to a proper RC circuit which cancels the effect of self inductance (Fig. 31). This is also called the "lossless inductor current sensing method". At low frequencies, the impedance of the RC network is much higher than the inductor, the capacitor current Ic(s) will be negligible and the inductor current $I_{L}(s)$ can be approximated to $I(s)$. Since $R>>r$ and $L_{C} \approx 0$, the transfer function between the capacitor voltage and the current can be approximated to

$$
\frac{V_{C}(s)}{I(s)} \approx \frac{V_{C}(s)}{I_{L}(s)}=\frac{L s+r}{R C s+1}
$$


If the $\mathrm{RC}$ network is chosen such that $r=\mathrm{L} / \mathrm{RC}$, the capacitor voltage will be proportional to the inductor current. The main problem concerns the temperature variations of $r$ and of the component values. This can be compensated by a simple scheme using a thermistor, and 1\% accuracy is achievable with this correction in a temperature range from $20{ }^{\circ} \mathrm{C}$ to $100^{\circ} \mathrm{C}$ [2009 Tsang]. However, there is still a variation of L with the DC current level, which is caused by the dependence of the core permeability on the core flux density. Ziegler [2009b] proposed a coupled sense winding method: the voltage induced in an extra compensation winding which is wound closely together with the main winding but which bears no current. If the two windings are identical, the voltage difference is proportional to the current. With carefully designed geometry, the corner frequency of such a sensor can reach $5 \mathrm{kHz}$.

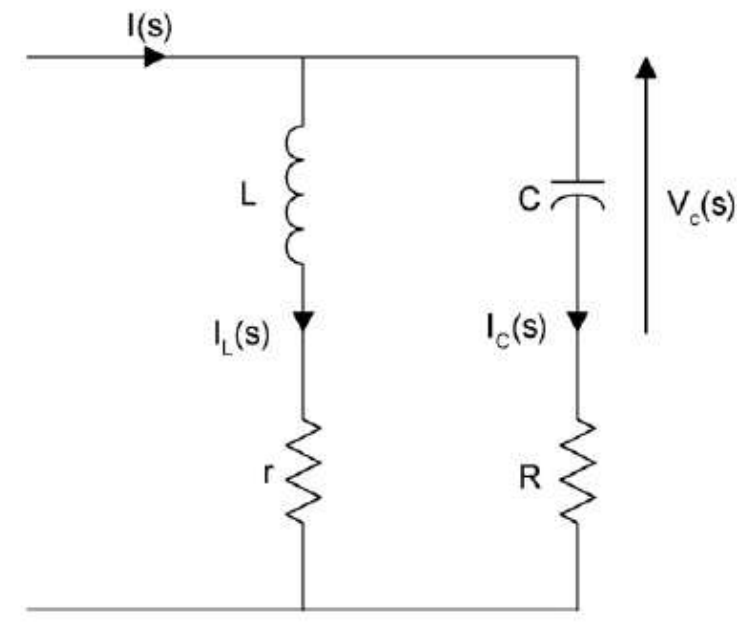

Fig. 31 from [Tsang 2009], reproduced with permission from Elsevier

\section{Current clamps}

Current clamps usually have an openable magnetic yoke, so they can be mounted without interrupting the measured conductor. The role of the yoke is again to concentrate the field lines so that the sensor reading is not dependent on the actual position of the clamped conductor and the device is insensitive to unclamped conductors.

AC current clamps are usually based on current transformer principles. The measured conductor forms the primary winding, and the multi-turn secondary winding is terminated by a small frequency-independent resistor, which is called the "burden". Alternatively, the secondary winding is connected to a current-tovoltage converter. Very accurate clamp current transformers use electronic compensation of the magnetization current and achieve errors of 0.05\% from the measured value in 1\% FS to 100\% FS. High-current $\mathrm{AC}$ and $\mathrm{AC} / \mathrm{DC}$ openable current transformer clamps [So and Bennet, 1993] and a low-current multistage clamp-on current transformer with ratio errors below 50 ppm [So and Bennet, 1997] have been developed.

DC current clamps are usually based on a Hall sensor in the airgap. These devices may have $10 \mathrm{~mA}$ resolution, but the maximum achieved accuracy is typically $30 \mathrm{~mA}$, even if they are of the compensated type. Their main disadvantage is unwanted sensitivity to external fields, due to the airgap in the magnetic circuit which is necessary for the Hall sensor. Even the change of position with respect to the Earth's field causes significant error, and the offset should be manually nulled.

Hall sensors in the airgap cannot be replaced by magneto resistors: although AMR sensors are more sensitive and stable, they are sensitive in the direction of the chip plane. They would therefore require a larger airgap (about $2 \mathrm{~mm}$ minimum), which degrades the sensor linearity and the geometrical selectivity. 
Precise DC/AC current clamps based on a shielded fluxgate sensor were described in [Kejik 1996]. The device shown in Fig. 32 has a rectangular ferrite core consisting of two symmetrical L-shaped halves. The permalloy shielding decreases the effect of the residual airgap at the clamp joint. Single winding serves for the excitation (by $1 \mathrm{kHz}$ squarewave voltage), sensing (second harmonics in the excitation current) and feedback. The sensor linearity and hysteresis error is less than $0.3 \%$ of the $40 \mathrm{~A}$ full-scale. The noise is $10 \mu \mathrm{A} \mathrm{p}-\mathrm{p}$, and the long-term zero stability is $1 \mathrm{~mA}$. The main advantage of using symmetrical shielding, which covers the residual air gaps in the joining points of the core, is high suppression of external currents and fields due to the virtually zero airgap. A similar type of clamps is presently manufactured by Tektronix. Figs. 33 and 34 show the effect of external current for the sensor without shielding, with asymmetrical CI type shielding and with symmetrical shielding according to Fig. 32. The symmetrical shielding is clearly technically superior. It requires a sliding mechanism with additional clamping force instead of simple single-joint clamping, but this type of slightly more complex mechanical design has become standard for precision clamps.

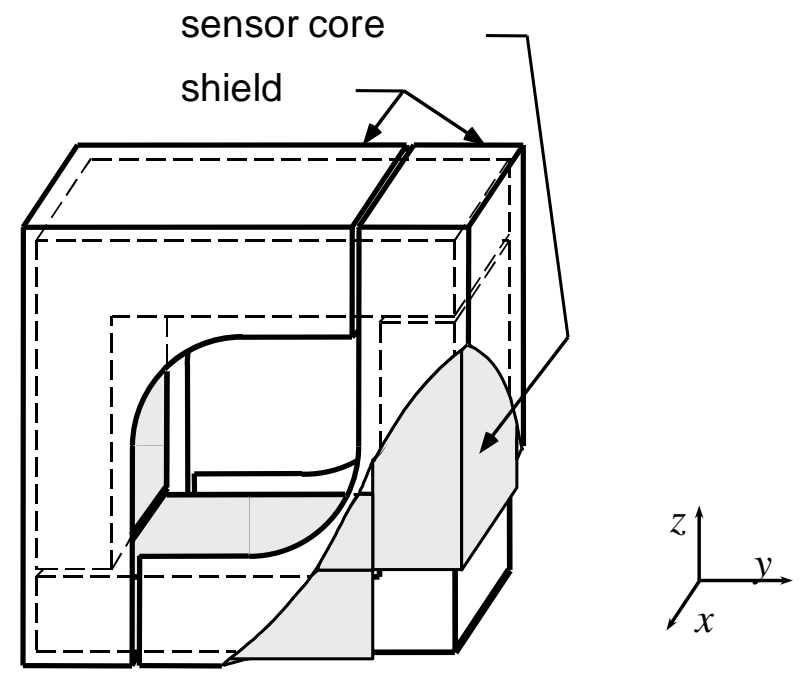

Fig. 32 - The shielded core of the current clamps - from [Kejik 1999] 


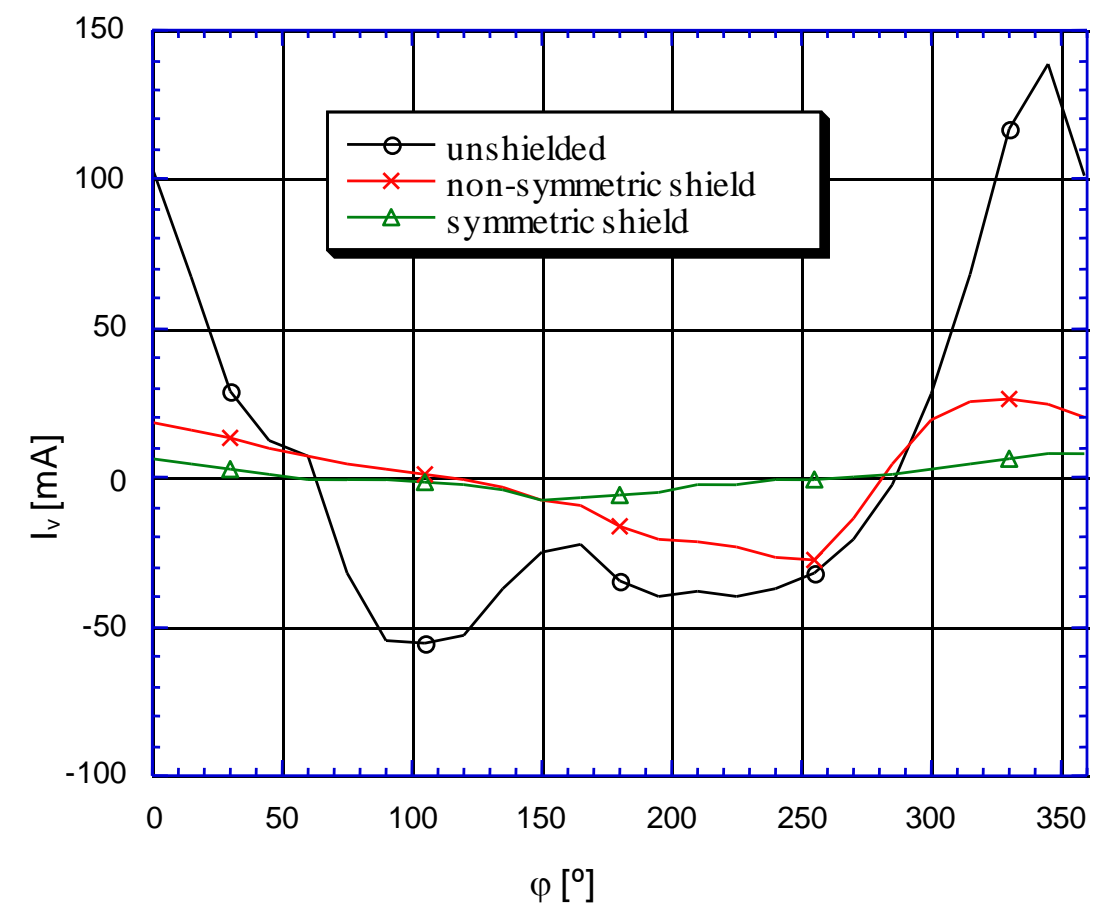

Fig. 33 Current error caused by external 40A current as a function of the "azimuth" position of the conductor in close vicinity of the current clamps from [Kejik 1996]

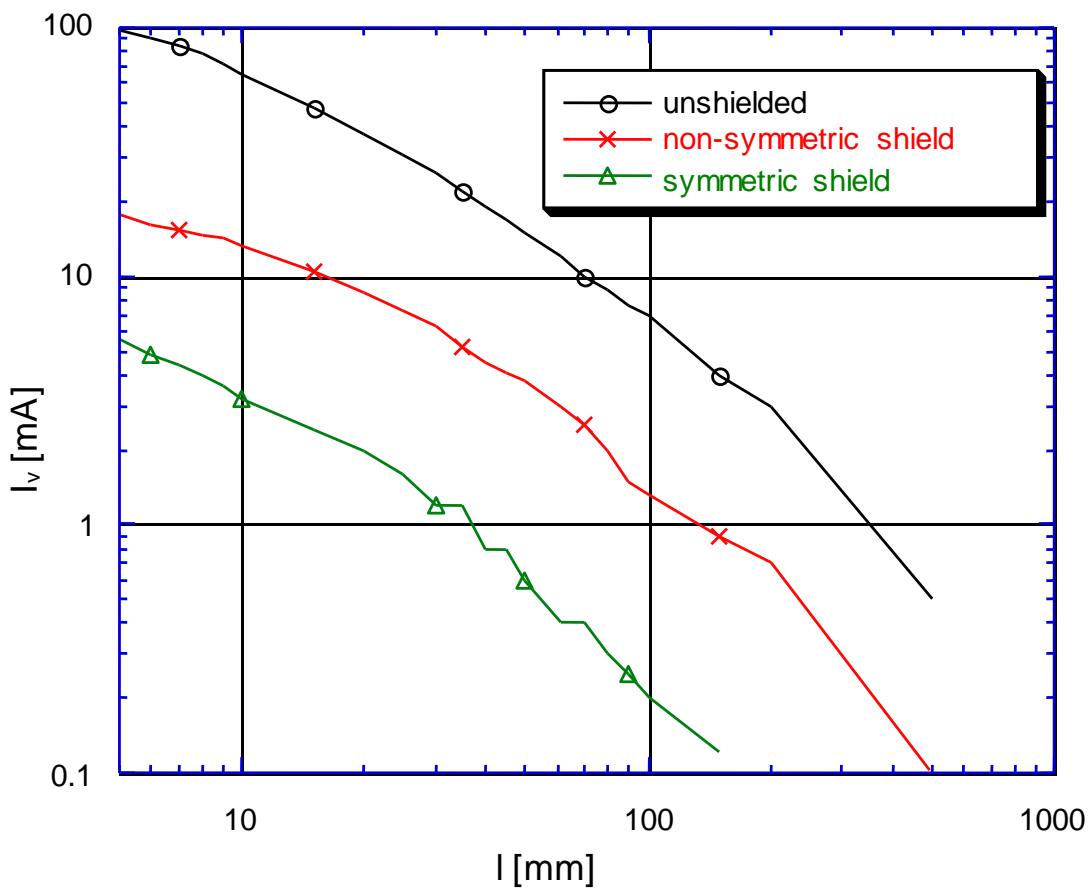

Fig. 34 Current error caused by external 40A current as a function of the conductor distance. Each measurement is made for the worst "azimuth" position from [Kejik 1996]

Very simple AC/DC current clamps can be improvised from AC commercially available current clamps, which are excited by an external AC generator into the fluxgate mode [Kejik 1999]. Two antiserially connected clamps were used to minimize the effect of the source impedance and AC interference injected into the measured 
circuit [Ripka 2004]. Two Iwatsu CP 502 AC oscilloscope current probes supplied by a $700 \mathrm{~Hz} / 290 \mathrm{~mA}$ p-p sinewave into serially connected secondary windings tuned by a parallel capacitor gave $11.6 \mathrm{mV} / \mathrm{A}$ sensitivity in the voltage-output mode and $300 \mathrm{~mA} / \mathrm{A}$ sensitivity in the current-output mode (per 1 turn of added detection winding).

Current clamps based on the fluxgate effect are used to permanently monitor the current flowing through the oil pipelines. For clamp diameters up to $33 \mathrm{~cm}, 1 \mathrm{~mA}$ resolution was achieved for a 2 A range, while for $150 \mathrm{~cm}$ diameter clamps the resolution was $10 \mathrm{~mA}$ in the 20 A range. The clamps have a maximum $40 \mathrm{~mA}$ response to the Earth's field (which can be nulled by proper installation), and achieved $15 \mathrm{~mA}$ offset stability through 5-year operation [Swain 2004].

Current clamps are not easy to characterise: the results are strongly affected by the location of the primary conductor with respect to the airgap in the magnetic circuit. Errors measured under non-sinusoidal conditions can be higher than those determined by the frequency response. This topic is analyzed in depth by [Cataliotti 2008].

Magneto optical current clamps are described in [Yi 2001]. They use bulk-optic glass, and not optical fibres. The achieved accuracy was 1\% for a $50 \mathrm{~Hz} A C$ current in a 1000 A range; the sensitivity was $4.45 \times 10^{-5} \mathrm{rad} / \mathrm{A}$, which is double the Verdet constant of SF-6 glass. Magneto optical clamps of another type use an openable magnetic circuit with an optical sensor in the airgap [Mihailovic 2004]. Errors caused by the displacement conductor range from $+1 \%$ in the vicinity of the magneto optical crystal to -3.7 in the opposite part the yoke, close to the openable gap.

No only traditional clamps but also flexible sensors can form an openable magnetic circuit. The most popular are flexible Rogowski coils (Section 4.2). A fluxgate current sensor with a flexible core made of superparamagnetic powder embedded in a flexible plastic matrix was reported in [Vourc'h 2009]. The sensor range is +/- $10 \mathrm{kA}$.

\section{Discussion and Conclusions}

Shunt resistors are still widely used, but they are very large and powerconsuming for high currents.

Traditional DC and AC contactless current sensors are available for ranges from $\mathrm{mA}$ to $\mathrm{kA}$ with precision from 3\% (uncompensated Hall current sensors) to $0.1 \%$ (compensated Hall devices and magnetic amplifiers). Higher precision is easily achievable with current comparators. Current sensors based on the AMR effect are very promising. If the measured current does not have a DC component, current transformers and di/dt sensors (Rogowski coils) are the best choice.

For $\mathrm{kA}$ and larger currents, Faraday Effect current sensors are attractive, as they are smaller and lighter than traditional sensors and they also have significantly lower power consumption. For smaller currents, optical sensors are not competitive with other sensor types: they are expensive, and they would require many turns of the fibre cable to achieve the required sensitivity.

\section{Acknowledgements}

The author thanks to many colleagues for valuable comments to this manuscript, mainly to D.A. Jackson, P. Kaspar, P. Mlejnek, R. Healey and K. Draxler. 
References

BIPM: SI Brochure

http://wWw.bipm.org/utils/common/pdf/si brochure 8 en.pdf

Appendix 2: http://www.bipm.org/en/si/si brochure/appendix2/electrical.html

W. Koon, Current Sensing for Energy Metering,

http://www .analog.com/Analog Root/static/technology/dataConverters/training/meter Background/currentSensors/Current sensing for metering.pdf 2009

Abdi-Jalebi, E. \& McMahon, R. 2007, "High-Performance Low-Cost Rogowski Transducers and Accompanying Circuitry", Instrumentation and Measurement, IEEE Transactions on, vol. 56, no. 3, pp. 753-759.

Alasia, D. \& Th\'evenaz, L. 2004, "A novel all-fibre configuration for a flexible polarimetric current sensor", Measurement Science and Technology, vol. 15, no. 8, pp. 1525-1530.

Baccigalupi, A. \& Liccardo, A. 2009\}, "Low-Cost Prototype for the Electronically Compensation of Current Transformers", IEEE SENSORS JOURNAL\}, vol. 9\}, no. 5-6\}, pp. 641-647\}.

Bazzocchi, R. \& Di Rienzo, L. 2000\}, "Interference rejection algorithm for current measurement using magnetic sensor arrays", SENSORS AND ACTUATORS APHYSICAL $\}$ vol. 85$\}$, no. 1-3\}, pp. 38-41\}.

Belloy, E., Gilbert, S., Dezuari, O., Sancho, M. \& Gijs, M. 2000\}, "A hybrid technology for miniaturised inductive device applications", SENSORS AND ACTUATORS A-PHYSICAL\}, vol. 85\}, no. 1-3\}, pp. 304-309\}.

Beltran, H., Reig, C., Fuster, V., Ramirez, D. \& Cubells-Beltran, M.D. 2007, "Modeling of Magnetoresistive-Based Electrical Current Sensors: A

Technological Approach", Sensors Journal, IEEE, vol. 7, no. 11, pp. 15321537 .

Bera, S. \& Chattopadhyay, S. 2003\}, "Study of an accurate dc busbar current measurement technique by using a transductor based network", MEASUREMENT\}, vol. 34$\},$ no. 3\}, pp. 255-261\}.

[Bergoz] WWW.bergoz.com

Beyer, J. \& Drung, D. 2008, "A SQUID series array dc current sensor", Superconductor Science and Technology, vol. 21, no. 9, pp. 095012 (6pp).

Bohnert, K., Brandle, H., Brunzel, M.G., Gabus, P. \& Guggenbach, P. 2007, "Highly Accurate Fiber-Optic DC Current Sensor for the Electrowinning Industry", Industry Applications, IEEE Transactions on, vol. 43, no. 1, pp. $180-187$.

Brandolini, A., Faifer, M. \& Ottoboni, R. 2009\}, "A Simple Method for the Calibration of Traditional and Electronic Measurement Current and Voltage Transformers", IEEE TRANSACTIONS ON INSTRUMENTATION AND MEASUREMENT\}, vol. $58\}$, no. 5\}, pp. 1345-1353\}.

Bull, J.D., Jaeger, N.A.F. \& Rahmatian, F. 2005, "A new hybrid current sensor for high-voltage applications", Power Delivery, IEEE Transactions on, vol. 20, no. 1, pp. 32-38.

CAMPBELL, W. \& ZIMMERMAN, J. 1980\}, "INDUCED ELECTRIC CURRENTS IN THE ALASKA OIL PIPELINE MEASURED BY GRADIENT FLUXGATE AND SQUID MAGNETOMETERS", IEEE 
TRANSACTIONS ON GEOSCIENCE AND REMOTE SENSING , vol. 18$\}$, no. 3\}, pp. $244-$ $250\}$.

Castaldo, F.C., Mognon, V.R. \& dos Reis Filho, C.A. 2009, "Magnetically Coupled Current Sensors Using CMOS Split-Drain Transistors", Power Electronics, IEEE Transactions on, vol. 24, no. 7, pp. 1733-1736.

Cataliotti, A., Di Cara, D., Emanuel, A.E. \& Nuccio, S. 2009\}, "Characterization of Clamp-On Current Transformers Under Nonsinusoidal Conditions", IEEE TRANSACTIONS ON POWER DELIVERY\}, vol. 24\}, no. 1\}, pp. 373$380\}$.

Cheng, C.H., Chen, M.H. \& Liu, W.F. 2007, "Measurement of AC current using an optical fibre sensor", physica status solidi (c), vol. 4, no. 12, pp. 45444547 .

Cimino, M., Lapuyade, H., De Matos, M., Taris, T., Deval, Y. \& Begueret, J.B. 2007\}," A robust 130 nm-CMOS built-in current sensor dedicated to RF applications", JOURNAL OF ELECTRONIC TESTING-THEORY AND APPLICATIONS\}, vol. $23\}$, no. 6\}, pp. 593-603\}.

Colin, B., Chillet, C., Kedous-Lebouc, A. \& Mas, P. 2006, "Effects of magnetic core geometry on false detection in residual current sensor", Journal of Magnetism and Magnetic Materials, vol. 304, no. 2, pp. e804.

Cruden A., I. Madden, C. Michie, P. Niewczas, J.R. McDonanld, I. Andonovic: Optical current measurement system for high-voltage applications, Measurement 24 , 97 (1998)

Dalessandro, L., Karrer, N. \& Kolar, J.W. 2007, "High-Performance Planar Isolated Current Sensor for Power Electronics Applications", Power Electronics, IEEE Transactions on, vol. 22, no. 5, pp. 1682-1692.

D'Antona, G., Di Rienzo, L., Ottoboni, R. \& Manara, A. 2001$\}$, "Processing magnetic sensor array data for AC current measurement in multiconductor systems", IEEE TRANSACTIONS ON INSTRUMENTATION AND MEASUREMENT\}, vol. 50\}, no. 5\}, pp. 1289-1295\}.

Deng, X., Li, Z., Peng, Q., Liu, J.\& Tian, J. 2008, "Research on the magneto-optic current sensor for high-current pulses", Review of Scientific Instruments, vol. 79, no. 8, pp. 083106-083106-4. 0034-6748

Di Rienzo, L., Bazzocchi, R. \& Manara, A. 2001\}, "Circular arrays of magnetic sensors for current measurement", IEEE TRANSACTIONS ON INSTRUMENTATION AND MEASUREMENT \}, vol. 50\}, no. 5\}, pp. 1093-1096\}.

Didosyan, Y.S., Hauser, H., Nicolics, J., Barash, V.Y. \& Fulmek, P.L. 2000, "Magneto-optical current sensor by domain wall motion in orthoferrites", Instrumentation and Measurement, IEEE Transactions on, vol. 49, no. 1, pp. $14-18$.

Dinev, P.D. 1996, "A two-dimensional remote fibre-optic magnetic field and current sensor", Measurement Science and Technology, vol. 7, no. 9, pp. 12331237 .

Donoval, M., Daricek, M., Stopjakova, V. \& Donoval, D. 2008 , "Magnetic fetbased on-chip current sensor for current testing of low-voltage circuits", JOURNAL OF ELECTRICAL ENGINEERING-ELEKTROTECHNICKY CASOPIS\}, vol. 59\}, no. $3\}$, pp. 122-130\}. 
Draxler, K. \& Styblikova, R. 1996\}, "Use of nanocrystalline materials for current transformer construction", JOURNAL OF MAGNETISM AND MAGNETIC MATERIALS , vol. 157\}, pp. 447-448\}.

Draxler, K. \& Styblikova, R. 2007\}, "Influence of instrument transformers on quality of electrical power and energy measurement", 2007 IEEE INTERNATIONAL SYMPOSIUM ON INDUSTRIAL ELECTRONICS, PROCEEDINGS, VOLS 1-8\}, pp. 1317.

Drexler, P. \& Fiala, P. 2008\}, "Utilization of Faraday Mirror in Fiber Optic Current Sensors", RADIOENGINEERING\}, vol. 17\}, no. 4$\}$.

Drung, D., Beyer, J., Peters, M., Storm, J.-. \& Schurig, T. 2009, "Novel SQUID Current Sensors With High Linearity at High Frequencies", Applied Superconductivity, IEEE Transactions on, vol. 19, no. 3, pp. $772-777$.

DURET, D., BERANGER, M. \& MOUSSAVI, M. 1992\}, "A NEW CURRENT SENSOR USING ELECTRON-SPIN-RESONANCE", SENSORS AND ACTUATORS A-PHYSICAL\}, vol. 31\}, no. 1$3\}$, pp. 174-177\}.

Edelstein, A. 2007\}, "Advances in magnetometry", JOURNAL OF PHYSICS-CONDENSED MATTER\}, Vol. 19$\}$, no. 16$\}$.

Ferrari, J.A., Flores, J.L., Dultz, W. \& Frins, E. 2009\}, "Optical current and voltage sensor using differential spectroscopy", OPTICAL ENGINEERING\}, vol. 48$\}$, no. 5$\}$.

Filipski, P.S. \& Boecker, M. 2006\}, "AC-DC current shunts and system for extended current and frequency ranges", IEEE TRANSACTIONS ON INSTRUMENTATION AND MEASUREMENT\}, vol. 55\}, no. 4\}, pp. 1222-1227\}.

Filipski, P.S., Boecker, M. \& Garcocz, M. 2008\}, "20-A to 100-A AC-DC Coaial current shunts for $100-\mathrm{kHz}$ frequency range", IEEE TRANSACTIONS ON INSTRUMENTATION AND MEASUREMENT\}, vol. 57\}, no. 8\}, pp. 1637-1641\}.

Fisher, N.E. \& Jackson, D.A. 1996a, "Vibration immunity and Ampere's circuital law for a near perfect triangular Faraday current sensor", Measurement Science and Technology, vol. 7, no. 8, pp. 1099-1102.

Fisher, N.E. \& Jackson, D.A. 1996b, "A common-mode optical noise-rejection scheme for an extrinsic Faraday current sensor", Measurement Science and Technology, vol. 7, no. 5, pp. 796-800.

Fisher, N.E. \& Jackson, D.A. 1995, "Improving the sensitivity of a Faraday current sensor by varying its operating point", Measurement Science and Technology, vol. 6, no. 10, pp. 1508-1518.

Fisher, N., Henderson, P. \& Jackson, D. 1997\}, "The interrogation of a conventional current transformer using an in-fibre Bragg grating", MEASUREMENT SCIENCE \\& TECHNOLOGY\}, vol. 8\}, no. 10\}, pp. 1080-1084\}.

Frick, V., Poure, P., Hebrard, L., Anstotz, F. \& Braun, F. 2007\}, "Electromagnetically compatible CMOS auto-balanced current sensor for highly integrated power control System-On-Chip", INTERNATIONAL JOURNAL OF ELECTRONICS \}, vol. 94$\}$, no. 1\}, pp. 9-22\}.

Fujiyama, Y., Yamada, Y., Kikuchi, H., Yamaguchi, M. \& Arai, K. 1997\}, "A fail-safe thin-film current sensor using a flux saturable ring core", IEEE TRANSACTIONS ON MAGNETICS\}, vol. 33\}, no. 5, Part 1\}, pp. 3406-3408\}. 
Gallo, D., Landi, C. \& Luiso, M. 2009\}, "COMPENSATION OF CURRENT TRANSFORMERS BY MEANS OF FIELD PROGRAMMABLE GATE ARRAY", METROLOGY AND MEASUREMENT SYSTEMS \}, vol. 16$\}$, no. 2\}, pp. 279-288\}.

Gay, F., Piquemal, F. \& Geneves, G. 2000, "Ultralow noise current amplifier based on a cryogenic current comparator", Review of Scientific Instruments, vol. 71, no. 12, pp. 4592-4595.

Gotz, M., Drung, D., Pesel, E., Barthelmess, H.-J., Hinnrichs, C., Assmann, C., Peters, M., Scherer, H., Schumacher, B., Schurig, T., 2009, Instrumentation and Measurement, IEEE Transactions on title=\{Improved Cryogenic Current Comparator Setup With Digital Current Sources, vol. 58, no. 4, pp. 1176 .

Han, B., Zhang, T., Huang, D., Yue, X., Zhou, Y. \& Bi, M. 2009, "Giant Magnetoimpedance Current Sensor With Spiral Structure Double-Probe", Magnetics, IEEE Transactions on, vol. 45, no. 4, pp. 1999-2002.

Hartmann, C., Mertin, W. \& Bacher, G. 2005\}, "Contactless current measurements using a needle sensor", ULTRAMICROSCOPY\}, vol. 105\}, no. 1-4\}, pp. $228-232\}$.

Iwansson, K., Sinapius, G. \& Hoornaert, W. 1999, Measuring Current, Voltage and Power, Elsevier.

Jackson, D.A. 2009\}, "An optical system with potential for remote health monitoring of subsea machinery", MEASUREMENT SCIENCE \\& TECHNOLOGY\}, vol. $20\}$, no. 3$\}$.

Keithley Inc.: Low Current Measurements, AN 100, 2007, available at www.keithley.com

Keithley Inc.: Low Level Measurements Handbook,6th edition, 2004 , available at www.keithley.com

Kejik, P., Ripka, P., Kaspar, P. \& Draxler, K. 1996, "Precise DC current sensors", Proc. IMTC, pp. 1479.

P. Kejik, Contactless measurement of currents and current ratio by fluxgate method, PhD thesis (in Czech), Czech Technical University, 1999. A pdf version can be obtained from the author (pavel.kejik@epfl.ch) or from the library of the Czech Technical University

Kim, H., Kang, G. \& Nam, D. 2009\}, "Coreless Hall Current Sensor for Automotive Inverters Decoupling Cross-coupled Field", JOURNAL OF POWER ELECTRONICS \}, vol. 9\}, no. 1\}, pp. 68-73\}. 1756-638X

Kim, J. \& Hong, S. 2006\}, "A CMOS built-in current sensor for IDDQ testing", IEICE TRANSACTIONS ON ELECTRONICS\}, vol. E89C\}, no. 6\}, pp. 868-870\}. 09168524

Kojovic L.: High-Precision Rogowski Coils for Improved Relay Protection, Control and Measurements, The Line, November 2002, pp. 7-8 available at http://www. cooperpower.com/Library/pdf/02049.pdf

Koga, F., Tadatsu, T., Inoue, J. \& Sasada, I. 2009\}, "A New Type of Current Sensor Based on Inverse Magnetostriction for Large Current Detection", IEEE TRANSACTIONS ON MAGNETICS\}, vol. 45\}, no. 10, Sp. IsS. SI\}, pp. 4506-4509\}. 
Kondrath, N. \& Kazimierczuk, M.K. 2009\}, "Bandwidth of Current Transformers", IEEE TRANSACTIONS ON INSTRUMENTATION AND MEASUREMENT\}, vol. 58\}, no. 6$\},$ pp. $2008-2016\}$.

Koon William, Current sensing for energy metering, , Analog Devices, Inc. www. analog. com

Kurosawa, K., Yamashita, K., Sowa, T. \& Yamada, Y. 2000\}, "Flexible fiber faraday effect current sensor using flint glass fiber and reflection scheme", IEICE TRANSACTIONS ON ELECTRONICS\}, vol. E83C\}, no. 3\}, pp. 326-330\}.

Lee, M.L., Park, J.S., Lee, W.J., Yun, S.H., Lee, Y.H. \& Kim, B.Y. 1998, "A polarimetric current sensor using an orthogonally polarized dual-frequency fibre laser", Measurement Science and Technology, vol. 9, no. 6, pp. 952-959.

Leland, E.S., Wright, P.K. \& White, R.M. 2009\}, "A MEMS AC current sensor for residential and commercial electricity end-use monitoring", JOURNAL OF MICROMECHANICS AND MICROENGINEERING\}, Vol. 19\}, no. 9 \}.

Lemme Helmuth and Andreas P. Friedrich: The Universal Current Sensor, Sensors magazine, 2000, Iss. 5, p. 82, also at

http://archives. sensorsmag.com/articles/0500/82/index.htm

Li, G.D., Aspey, R.A., Kong, M.G., Gibson, J.R. \& Jones, G.R. 1999, "Elliptical polarization effects in a chromatically addressed Faraday current sensor", Measurement Science and Technology, vol. 10, no. 1, pp. 25-30.

Liang, Y.C., Samudra, G.S., Lim, A.J.D. \& Pick Hong Ong 2003, "Accurate current sensor for lateral IGBT smart power integration", Power Electronics, IEEE Transactions on, vol. 18, no. 5, pp. 1238-1243.

Lopez-Garcia, O., Carnicero, A., Ru\'iz, R., Talavera, J.A. \& Mateo, C. 2006, "Development of a current sensor based on active materials for high-voltage transmission systems", Smart Materials and Structures, vol. 15, no. 2, pp. $563-570$.

Lucas I., M. D. Michelena, R. P. del Real, V. de Manuel, J. A. Plaza, M. Duch, J. Esteve, and H. Guerrero: A New Single-Sensor Magnetic Field Gradiometer, SENSOR LETTERS Vol. 7, No. 4, 2009, pp. 1-8, doi:10.1166/sl.2009.1110

Malatek, M., Ripka, P. \& Kraus, L. 2005\}, "Double-core GMI current sensor", IEEE TRANSACTIONS ON MAGNETICS\}, vol. 41\}, no. 10\}, pp. 3703-3705\}.

[Melexis] http://www.melexis.com/

Malatek, M., Ripka, P. \& Kraus, L. 2008\}, "Temperature offset drift of GMI sensors", SENSORS AND ACTUATORS A-PHYSICAL\}, vol. 147\}, no. 2\}, pp. 415-418\}.

Miljanic P.N., So E., and Moore W.J.M, An electronically enhanced magnetic core for current transformers, IEEE Trans. Instrum. and Meas., Vol. 40, 1991, pp. 410414

Mlejnek, P., Kaspar, P. \& Draxler, K. 2007\}, "Measurement of ratio error and phase displacement of DC tolerant current transformers", SENSOR LETTERS , vol. 5\}, no. 1\}, pp. 289-292\}.

Mlejnek, P., Vopalensky, M. \& Ripka, P. 2008\}, "AMR current measurement device", SENSORS AND ACTUATORS A-PHYSICAL\}, vol. 141\}, no. 2\}, pp. 649-653\}.

Mlejnek, P. - Kašpar, P.: Detection of Disturbance of Working Conditions of Current Transformer in Energy Meters for Reduction of Unauthorized Current 
Consumption In: PEDS 2009 - The Eighth International Conference on Power Electronics and Drive Systems [CD-ROM]. Taipei: IEEE Power Electronics Society - PELS, 2009 ,

[Mlejnek 2009b]

Mlejnek, P. \& Kaspar, P. 2009b\}, "Drawback of Using Double Core Current Transformers in Static Watt-Hour Meters", SENSOR LETTERS\}, vol. 7\}, no. 3, Sp. IsS. SI\}, pp. 394-396\}.

Moore, W.J.M. \& Miljanic, P.N.: The Current Comparator, 1998, Peter Peregrinus, London.

Ning, Y.N., Wang, Z.P., Palmer, A.W. \& Grattan, K.T.V. 1995, "A Faraday current sensor using a novel multi-optical-loop sensing element", Measurement Science and Technology, vol. 6, no. 9, pp. 1339-1342.

Northrop, R.B. 2005, Introduction to Instrumentation and Measurement, Taylor and Francis CRC.

O'Donnell, T., Tipek, A., Connell, A., McCloskey, P. \& O'Mathuna, S. 2006\}, "Planar fluxgate current sensor integrated in printed circuit board", SENSORS AND ACTUATORS A-PHYSICAL\}, vol. 129\}, no. 1-2, Sp. IsS. SI\}, pp. 20-24\}.

Pannetier-Lecoeur, M., Fermon, C., de Vismes, A., Kerr, E. \& Vieux-Rochaz, L. 2007\}, "Low noise magnetoresistive sensors for current measurement and compasses", JOURNAL OF MAGNETISM AND MAGNETIC MATERIALS\}, vol. 316\}, no. 2\}, pp. E246-E248\}.

Pastre, M., Kayal, M. \& Blanchard, H. 2007\}, "A Hall sensor analog front end for current measurement with continuous gain calibration", IEEE SENSORS JOURNAL\}, vol. 7\}, no. 5-6\}, pp. 860-867\} .

Perciante, C.D. \& Ferrari, J.A. 2008, "Magnetic Crosstalk Minimization in Optical Current Sensors", Instrumentation and Measurement, IEEE Transactions on, vol. 57, no. 10, pp. 2304-2308.

Ponjavic, M.M. \& Duric, R.M. 2007, "Nonlinear Modeling of the SelfOscillating Fluxgate Current Sensor", Sensors Journal, IEEE, vol. 7, no. 11, pp. 1546-1553.

Popovic, R., Drljaca, P. \& Kejik, P. 2006\}, "CMOS magnetic sensors with integrated ferromagnetic parts", SENSORS AND ACTUATORS A-PHYSICAL\}, vol. $129\}$, no. 1-2, Sp. Iss. SI\}, pp. 94-99\}.

Qing, C., Hong-bin, L., Ben-xiong, H. \& Qiao-qi, D. 2009\}, "Rogowski Sensor for Plasma Current Measurement in J-TEXT", IEEE SENSORS JOURNAL\}, vol. 9\}, no. 3$\}$, pp. 293-296\}.

Qiu, D.Y., Yip, S.C., Chung, H.S.-. \& Hui, S.Y.R. 2003, "On the use of current sensors for the control of power converters", Power Electronics, IEEE Transactions on, vol. 18, no. 4, pp. 1047-1055.

Reig, C., Ramirez, D., Silva, F., Bernardo, J. \& Freitas, P. 2004\}, "Design, fabrication, and analysis of a spin-valve based current sensor", SENSORS AND ACTUATORS A-PHYSICAL\}, vol. 115\}, no. 2-3\}, pp. 259-266\}.

Reilly, D., Willshire, A.J., Fusiek, G., Niewczas, P. \& McDonald, J.R. 2006\}, "A fiber-Bragg-grating-based sensor for simultaneous AC current and temperature measurement", IEEE SENSORS JOURNAL\}, vol. 6\}, no. 6\}, pp. 1539$1542\}$. 
Ribeiro, R.M. \& Werneck, M.M. 2008\}, "Improvements of resolution and precision of a wavelength-encoded electrical current sensor using an ultrabright light-emitting diode transducer", TRANSACTIONS OF THE INSTITUTE OF MEASUREMENT AND CONTROL\}, vol. 30\}, no. 2\}, pp. 153-171\}.

Ripka, P. (ed.), Magnetic sensors, Artech 2001

Ripka, P. 2004\}, "Current sensors using magnetic materials", JOURNAL OF OPTOELECTRONICS AND ADVANCED MATERIALS\}, vol. 6\}, no. 2\}, pp. 587-592\}.

Ripka, P., Kubik, J., Duffy, M., Hurley, W. \& O'Reilly, S. 2005\}, "Current sensor in PCB technology", IEEE SENSORS JOURNAL\}, vol. 5\}, no. 3\}, pp. 433$438\}$.

[Sensitec] www.sensitec.com

SCOVILLE, J. \& PETERSEN, P. 1991$\}$, "A LOW-COST MULTIPLE HALL PROBE CURRENT TRANSDUCER", REVIEW OF SCIENTIFIC INSTRUMENTS\}, vol. 62\}, no. 3\}, pp. 755$760\}$.

So, E. \& Bennett, D. 1997\}, "A low-current multistage clamp-on current transformer with ratio errors below 50x10(-6)", IEEE TRANSACTIONS ON INSTRUMENTATION AND MEASUREMENT\}, Vol. 46\}, no. 2\}, pp. 454-458\}.

SO, E., REN, S. \& BENNETT, D. 1993\}, "HIGH-CURRENT HIGH-PRECISION OPENABLECORE AC AND AC DC CURRENT TRANSFORMERS", IEEE TRANSACTIONS ON INSTRUMENTATION AND MEASUREMENT\}, vol. 42\}, no. 2\}, pp. 571-576\}.

Souders T. M.: Wide-Band Two-Stage Current Transformers of High Accuracy, IEEE Trans. Instrum. Meas. IM-21, 340 (1972).

Suomalainen, E. \& Hallstrom, J.K. 2009\}, "Onsite Calibration of a Current Transformer Using a Rogowski Coil", IEEE TRANSACTIONS ON INSTRUMENTATION AND MEASUREMENT\}, vol. 58\}, no. 4\}, pp. 1054-1058\}.

Steiner, R. L.;Williams, E. R.; Liu, R.; Newell, D. B. (2007), Uncertainty Improvements of the NIST Electronic Kilogram IEEE Trans. Instrum. Meas. 56 (2) : 592

Swain, W. 2004\}, "A permanently buried clamp-on direct current ammeter sensor", MATERIALS PERFORMANCE\}, vol. 43\}, no. 2\}, pp. 20-22\}.

Tang, S., Duffy, M., Ripka, P. \& Hurley, W. 2004\}, "Excitation circuit for fluxgate sensor using saturable inductor", SENSORS AND ACTUATORS A-PHYSICAL\}, vol. 113$\}$, no. 2$\}$, pp. 156-165\}.

Takagi S., Kojima J., and Asakawa K., "DC cable sensors for locating underwater telecomunication cables", proc. IEEE OCEANS 96 conf., New York 1996 , pp. 339-344

Takahashi, M., Sasaki, K., Ohno, A., Hirata, Y. \& Terai, K. 2004 , "Sagnac interferometer-type fibre-optic current sensor using single-mode fibre down leads", Measurement Science and Technology, vol. 15, no. 8, pp. 1637-1641.

Tsang, K.M. \& Chan, W.L. 2009, "Temperature compensated high efficiency inductor current sensor", Sensors and Actuators A: Physical, vol. 154, no. 1, pp. 16 .

Unser, K.B. 1981, "A toroidal DC beam current transformer with high resolution", IEEE TRANSACTIONS ON NUCLEAR SCIENCE\}, vol. NS-28, pp. 23442346 . 
Unser, K.B. 1991, "The parametric current transformer, a beam current monitor developed for LEP. oai:cds.cern.ch:231598", 3rd Annual Workshop on Accelerator Instrumentation, 28 - 31 Oct 1991, pp. 266.

Vazquez, J.R. \& Pineda de Gyvez, J. 2004, "Built-in current sensor for $\triangle I D D Q$ testing", Solid-State Circuits, IEEE Journal of, vol. 39, no. 3, pp. 511-518.

Vieth, M Clemens, W van den Berg, H Rupp, G Wecker, J Kroeker, M Contactless current detection with GMR sensors based on an artificial antiferromagnet (AAF) subsystem Sens. Act. A 81 (2000) pp. $44-48$

Vourc'h, E., Joubert, P.- \& Cima, L. 2009\}, "ANALYTICAL AND NUMERICAL ANALYSES OF A CURRENT SENSOR USING NON LINEAR EFFECTS IN A FLEXIBLE MAGNETIC TRANSDUCER", PROGRESS IN ELECTROMAGNETICS RESEARCH-PIER\}, vol. 99\}, pp. 323$338\}$.

Waeckerlé, T., Fraisse, H., Furnemont, Q. \& Bloch, F. 2006, "Upgrade Fe-50\%Ni alloys for open-loop DC current sensor: Design and alloy-potential

characteristics", Journal of Magnetism and Magnetic Materials, vol. 304, no. 2 , pp. e850.

Wang, Z.P., Wang, X., Liu, X., Ouyang, C. \& Tan, Q. 2005, "Effect of the spectral width of optical sources upon the output of an optical current sensor", Measurement Science and Technology, vol. 16, no. 8, pp. 1588-1592.

Wang, Z., Wang, H., Jiang, H. \& Liu, X. 2007\}, "A magnetic field sensor based on orthoconjugate reflection used for current sensing", OPTICS AND LASER TECHNOLOGY\}, vol. 39\}, no. 6\}, pp. 1231-1233\}.

Ward, D.A. \& Exxon, J.L.T. 1993, "Using Rogowski coils for transient current measurements", ENGINEERING SCIENCE AND EDUCATION JOURNAL, , no. June, pp. $105-113$.

Watanabe, T., Sasaki, Y., Kase, M., Watanabe, S., Ikeda, T., Kawaguchi, T. \& Yano, Y. 2008, "Development of new HTS-SQUID and HTS current sensor for HTSSQUID beam current monitor", Journal of Physics: Conference Series, vol. 97, pp. 012248 (6pp).

Weckenmann, A., Hoffmann, J. \& Schuler, A. 2008, "Development of a tunnelling current sensor for a long-range nano-positioning device", Measurement Science and Technology, vol. 19, no. 6, pp. 064002 (7pp).

Wirth, G. 2008, "Bulk built in current sensors for single event transient detection in deep-submicron technologies", Microelectronics Reliability, vol. 48 , no. 5, pp. 710 .

Yi, B., Chu, B.C.B. \& Chiang, K.S. 2002, "Magneto-optical electric-current sensor with enhanced sensitivity", Measurement science and Technology, vol. 13, no. 7, pp. N61-N63.

Yi, B., Chu, B. \& Chiang, K. 2001\}, "New design of a detachable bulk-optic Faraday effect current clamp", OPTICAL ENGINEERING\}, vol. 40\}, no. 6\}, pp. $914-920\}$.

Yoshino, T., Minegishi, K. \& Nitta, M. 2001, "A very sensitive Faraday effect current sensor using a YIG/ring-core transformer in a transverse configuration", Measurement Science and Technology, vol. 12, no. 7, pp. 850853 .

Zhan, Z., Yaoming, L., Jin, C. \& Yunfeng, X. 2007\}, "Current sensor utilizing giant magneto-impedance effect in amorphous ribbon toroidal core and CMOS 
inverter multivibrator", SENSORS AND ACTUATORS A-PHYSICAL\}, vol. 137$\}$, no. $1\}$, pp. 64-67\}.

Zhao, Y., Meng, Q. \& Chen, K. 2006\}, "Novel current measurement method based on fiber Bragg grating sensor technology", SENSORS AND ACTUATORS A-PHYSICAL\}, vol. 126\}, no. 1\}, pp. 112-116\}.

Zhou, S. \& Zhang, X. 2007, "Simulation of Linear Birefringence Reduction in Fiber-optical Current Sensor", Photonics Technology Letters, IEEE, vol. 19, no. 19, pp. 1568-1570.

[Ziegler 2009a]

Ziegler, S., Woodward, R.C., Iu, H.H. \& Borle, L.J. 2009\}, "Current Sensing Techniques: A Review", IEEE SENSORS JOURNAL\}, vol. 9\}, no. 4\}, pp. 354-376\}.

[Ziegler 2009b].

Ziegler, S., Woodward, R.C., Iu, H.H.C. \& Borle, L.J. 2009\}, "Lossless Inductor Current Sensing Method With Improved Frequency Response", IEEE TRANSACTIONS ON POWER ELECTRONICS\}, VOI. 24\}, no. 5-6\}, pp. 1218-1222\}.

[Ziegler 2009c].

Ziegler, S., Woodward, R.C., Iu, H.H. \& Borle, L.J. 2009\}, "Investigation Into Static and Dynamic Performance of the Copper Trace Current Sense Method", IEEE SENSORS JOURNAL\}, vol. 9\}, no. 7\}, pp. 782-792\}. 